\title{
Insights into Acinetobacter baumannii: A Review of Microbiological, Virulence, and Resistance Traits in a Threatening Nosocomial Pathogen
}

\author{
Carole Ayoub Moubareck ${ }^{1, *,+}$ and Dalal Hammoudi Halat ${ }^{2,+}+($ ) \\ College of Natural and Health Sciences, Zayed University, Dubai P.O. Box 144534, UAE \\ 2 Department of Pharmaceutical Sciences, School of Pharmacy, Lebanese International University, Beirut, \\ Bekaa Campuses 1103, Lebanon; dalal.hammoudi@liu.edu.lb \\ * Correspondence: Carole.AyoubMoubareck@zu.ac.ae; Tel.: +971-4-402-1745 \\ $\dagger$ Authors contributed equally to the manuscript.
}

Received: 3 February 2020; Accepted: 2 March 2020; Published: 12 March 2020

\begin{abstract}
Being a multidrug-resistant and an invasive pathogen, Acinetobacter baumannii is one of the major causes of nosocomial infections in the current healthcare system. It has been recognized as an agent of pneumonia, septicemia, meningitis, urinary tract and wound infections, and is associated with high mortality. Pathogenesis in A. baumannii infections is an outcome of multiple virulence factors, including porins, capsules, and cell wall lipopolysaccharide, enzymes, biofilm production, motility, and iron-acquisition systems, among others. Such virulence factors help the organism to resist stressful environmental conditions and enable development of severe infections. Parallel to increased prevalence of infections caused by A. baumannii, challenging and diverse resistance mechanisms in this pathogen are well recognized, with major classes of antibiotics becoming minimally effective. Through a wide array of antibiotic-hydrolyzing enzymes, efflux pump changes, impermeability, and antibiotic target mutations, $A$. baumannii models a unique ability to maintain a multidrug-resistant phenotype, further complicating treatment. Understanding mechanisms behind diseases, virulence, and resistance acquisition are central to infectious disease knowledge about $A$. baumannii. The aims of this review are to highlight infections and disease-producing factors in A. baumannii and to touch base on mechanisms of resistance to various antibiotic classes.
\end{abstract}

Keywords: Acinetobacter baumannii; multidrug resistance; virulence factors; antibiotics; pathogenesis

\section{Introduction}

Over the last decades, Acinetobacter baumannii has globally emerged as a highly troublesome nosocomial pathogen. Its clinical significance has been largely driven by a remarkable ability to acquire or upregulate various resistance determinants, making it one of the most successful multidrug-resistant (MDR) organisms threatening current antibiotic therapy [1]. On top of such fascinating resistance acquisition, A. baumannii is endowed with multiple mechanisms of survival under a wide range of environments, potentiating capacity for hospital spread [2]. The attributable mortalities in patients with A. baumannii healthcare-associated infections, of which ventilator-associated pneumonia and bloodstream infections are the most common, can range from $5 \%$ in general hospital wards to $54 \%$ in the intensive care unit (ICU) [3], with increasing reports of community-acquired A. baumannii infections [4]. Mounting evidence of extensively drug-resistant (XDR) and pandrug-resistant (PDR) isolates of A. baumannii is also accumulating in different countries [5-7]. The World Health Organization (WHO) has assigned A. baumannii as a critical priority pathogen posing a great threat to human health, and towards which new antibiotics are urgently needed [8]. Such clinical and public health implications 
underlie the need to further understand and evaluate both disease and antibiotic resistance mechanisms in this pathogen. The aims of the current review are to highlight clinically relevant infections and disease-producing factors in A. baumannii, and to revise its mechanisms of resistance to various antibiotic classes.

\section{Taxonomy and Microbiological Properties of Acinetobacter baumannii}

The current definition of the genus Acinetobacter consists of short, pleomorphic coccobacilli that are Gram-negative, strictly aerobic, catalase-positive, oxidase-negative, nonfermenting, and nonmotile. Their DNA G+C content ranges between 39\% to $47 \%$. Acinetobacter produces at $37^{\circ} \mathrm{C}$ grayish-white, smooth, mucoid colonies on solid media commonly used for diagnostic purposes, like sheep blood agar and tryptic soy agar [2].

After its first description at the beginning of the 20th century, this heterogeneous group of bacteria has gone through a remarkable, complex, taxonomic history. Since the 1980s, and in correspondence to wide recognition and emergence of acinetobacters as nosocomial pathogens, refined taxonomy has been constantly updated [4]. Thanks to the work of Bouvet and Grimont [9], an initial landmark classification of acinetobacters was based on DNA-DNA hybridization studies, and distinguished 12 DNA groups or genospecies, some of which were given formal names like A. baumannii, Acinetobacter calcoaceticus, Acinetobacter haemolyticus, Acinetobacter johnsonii, Acinetobacter junii, and Acinetobacter lwoffii. As of 2019 and according to a review published by Vijayakumar et al., 59 species belong to the genus Acinetobacter, where 11 have defined names and 15 have a tentative description [10]. In particular, the Acinetobacter calcoaceticus-Acinetobacter baumannii complex (ACB complex) comprises four species: A. calcoaceticus (genomic species 1), A. baumannii (genomic species 2), Acinetobacter pittii (previously genomic species 3), and Acinetobacter nosocomialis (previously genomic species $13 \mathrm{TU}$ ) that are closely related and difficult to distinguish by phenotypic properties [11]. Recently, two new species, Acinetobacter seifertii and Acinetobacter dijkshoorniae, were also included within the ACB complex. Hence, the ACB complex collectively includes five Acinetobacter species associated with human diseases (A. baumannii, A. pittii, A. nosocomialis, A. seifertti, and A. dijkshoorniae) and one environmental species (A. calcoaceticus), which is commonly isolated from soil and has not been described as a human pathogen [12,13].

The identification of Acinetobacter to the species level remains complicated and challenging. Phenotypic methods based upon growth temperatures, hemolysis, glucose acidification, and carbon/energy sources [10], in addition to commercial automated systems [14], are proposed. Molecular species identification by DNA-DNA hybridization studies [15], 16S rRNA sequencing [16], and matrix-assisted laser desorption ionization-time of flight mass spectrometry (MALDI-TOF) [17] are increasingly being used. In addition, there is a transition from traditional typing methodology to whole-genome sequencing-based approaches that are proving their utility in epidemiological classification of Acinetobacter. Performance of such methods demonstrates superiority to conventional typing and promises useful future incorporation in infection control besides current use in epidemiological research [18,19]. A reliable biomarker for identification of the species A. baumannii is the $b l a_{\text {OXA-51-like }}$ gene, chromosomally located in this species and displaying very weak hydrolysis of carbapenems [20]. The utility of this gene for differentiation of A. baumannii from ACB complex has been elaborated by Turton and colleagues [21].

A. baumannii, $A$. pittii, and $A$. nosocomialis are still difficult to precisely identify in most laboratories and they represent the three most clinically relevant species of Acinetobacter that have been implicated in the vast majority of both community-acquired and nosocomial infections [4]. Hence, this review will use A. baumannii in the comprehensive sense to refer to these three species.

\section{Associated Infections and Clinical Impact of A. baumannii}

As mentioned above, A. baumanni has propensity to tolerate stressful environments and multiple classes of antibiotics, making it able to survive and spread as a nosocomial pathogen, particularly in critically ill patients, contributing to increased morbidity and mortality [22]. Previous studies 
addressing risk factors for acquisition of $A$. baumannii have reported multiple culprits including long ICU stay, previous hospital or ICU stay, previous antimicrobial therapy, mechanical ventilation, use of devices (indwelling catheters, endotracheal tube or nasogastric tube), older age, major or emergent surgery, low birth weight or prematurity, dialysis, and prolonged use of total parenteral nutrition or intravenous lipids [23-26]. In recent years, A. baumannii infections involving respiratory tract, bloodstream, skin and soft tissue, urinary tract, and central nervous system have emerged as highly problematic in healthcare institutions. Figure 1 summarizes A. baumannii disease burden, resistance, and endemicity in humans, animals, and the environment.

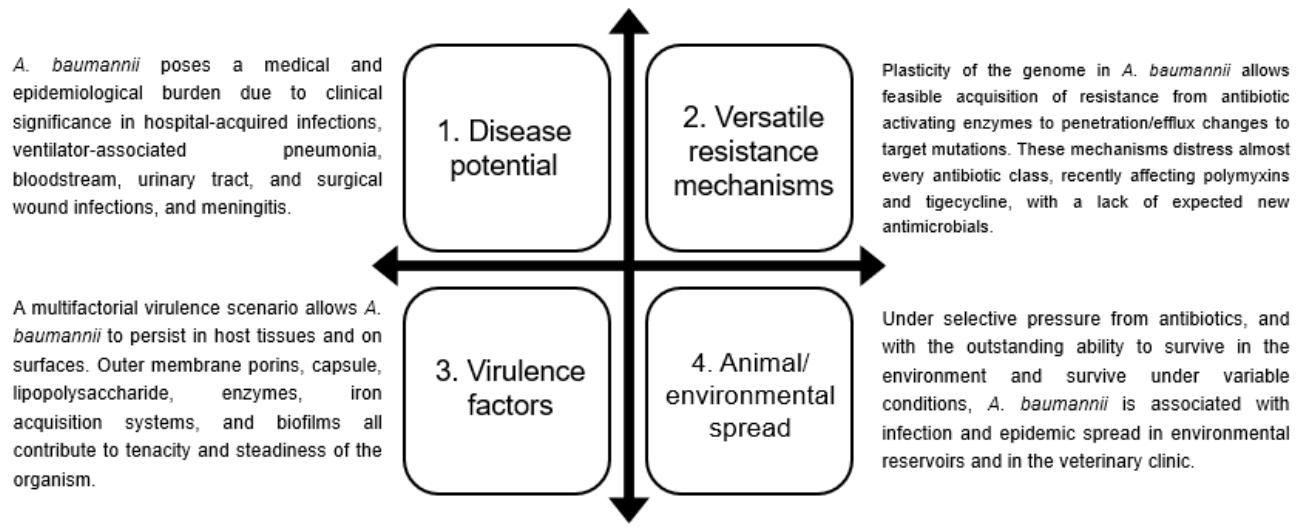

Figure 1. The dynamic microbiological nature of Acinetobacter baumannii derives from an interplay between the associated infections, wide arsenal of virulence factors, multidrug-resistant phenotype, and spread in animals and the environment.

\subsection{Respiratory Infections}

Ventilator-associated pneumonia (VAP) caused by MDR A. baumannii remains a leading cause of high mortality rate in critically ill patients [27]. A. baumannii accounts for $8 \%$ to $14 \%$ of VAP in the United States and Europe, but this pathogen is associated with higher rates (19\% to >50\%) in Asia, Latin America, and some Middle Eastern countries [28]. Recent research indicated that incidence of MDR A. baumannii transmission was 315.4 cases/1000 ICU patient-days with mortality rate ranging from $52 \%$ to $66 \%$ [29]. In a seven-year survey of MDR A. baumannii infections in a tertiary care center in Lebanon, the most common site of infection among the isolates was the respiratory tract (53.1\%), followed by surgical wound (18.8\%), blood (15.6\%), urine $(10.2 \%)$, and others $(2.3 \%)$. The most common colonization site was the respiratory tract (80.8\%) followed by skin colonization (12.4\%) [30]. In a more recent investigation from a university hospital, rates of MDR, XDR, and PDR A. baumannii recovered from VAP cases were 13.3\%, 68.3\%, and 18.3\%, respectively, with female gender and red blood cell transfusion as independent risk factors for mortality [31]. Furthermore, a predominant clonal lineage of $A$. baumannii was found to be associated with VAP across 12 hospital centers in three European countries, and suggests the emergence of a XDR/PDR A. baumannii epidemic, with resistant rates close to $100 \%$ for carbapenem and $50 \%$ for colistin [32]. In a recent meta-analysis involving 29 countries, the prevalence of MDR phenotype among A. baumannii causing hospital-acquired pneumonia and VAP was close to $80 \%$. Central America, Latin America, and the Caribbean had the highest prevalence, whereas Eastern Asia had the lowest [33]. Also, in a screening analysis for lower respiratory tract specimens of 97 patients infected with A. baumannii in Southern Vietnam, $80 \%$ of the specimens were carbapenem resistant and 90\% were MDR [34].

Although A. baumannii-induced VAP appears to have a predilection for vulnerable patients, community-acquired pneumonia (CAP) due to this organism is an increasing cause for concern. It is characterized by a fulminant course, high incidence of bacteremia, and high mortality rate, especially in tropical regions, where it predominantly affects individuals with excess alcohol consumption, diabetes mellitus, smoking, and chronic lung disease [35]. CAP caused by A. baumannii is prevalent in different 
parts of Australia, Oceania, and Asia, including Taiwan, China, and Thailand [36]. A recent Chinese study reported $A$. baumannii CAP caused by a rare sporadic clone of sequence type 880 that carries the plasmid-encoded $b l a_{\mathrm{OXA}-72}$ gene conferring resistance to carbapenems [37]. Accumulating scientific evidence about the role of $A$. baumannii in respiratory tract infections needs to be put in perspective for better surveillance and control.

\subsection{Bloodstream Infections}

A. baumannii has become a leading cause of bloodstream infections in health care settings with intravenous catheters or the respiratory tract representing a frequent source of infection. Mortality rate due to bloodstream infections caused by A. baumannii is approaching $40 \%$ [38]. High incidence of bacteremia due to $A$. baumannii has been reported in cancer patients, where this organism accounted for about $68 \%$ of bacteremia cases in a Brazilian study. Unfortunately, such bacteremia had a high mortality among cancer patients, and risk factors associated with infection-related mortality appeared to be more closely linked to management of bacteremia than to special characteristics of the cancer population, such as neutropenia and tumor site infection [39]. In another category of patients, namely those undergoing neurosurgery, about $13 \%$ of bloodstream infections over a period of 10 years were attributed to A. baumannii, of which $90 \%$ were carbapenem resistant [40]. In another study discussing severely burned patients admitted to a burn ICU, A. baumannii was the top isolated pathogen from blood for four consecutive years, and except for low resistance profile to polymyxin B and minocycline, the isolates were almost $100 \%$ resistant to other antibiotics [41]. In a recent study from a hospital in Northwest Ethiopia, A. baumannii accounted for about $9 \%$ of nosocomial bloodstream infections, and were $100 \%$ resistant to ampicillin and piperacillin, and $33.3 \%$ and $44.5 \%$ resistant to meropenem and ciprofloxacin, respectively [42]. Moreover, $42 \%$ of $A$. baumannii causing bloodstream infections in ICU patients from a Greek university hospital were resistant to colistin, and were directly linked to fulminant septic shock and high mortality [43]. Such wealth of data regarding the upsurge of drug-resistant $A$. baumannii in bloodstream infections, especially in severely ill patients, requires emphasis on surveillance and characterization of antimicrobial resistance patterns, as well as reinforcement of appropriate antibiotic and infection control measures.

\subsection{Skin and Soft Tissue Infections}

A. baumannii has been repeatedly isolated from skin and soft tissue in patients with severe burns, wounds, or trauma, for instance, soldiers injured during military operations or victims of natural disasters. A landmark paper by Davis and colleagues [44] reported war wound infection and osteomyelitis caused by MDR A. baumannii during the 2003-2005 military operations in Iraq. In another retrospective study of US military service members wounded in Iraq or Afghanistan, A. baumanniii was the most commonly isolated pathogen from open tibial fractures [45]. Afghanistan and Iraq remain the two major geographic locations where $A$. baumannii were isolated from wound or soft tissue especially after traumatic injury [36]. In a military medical center in the US, overall A. baumannii isolation increased from $4 \%$ to $55 \%$ over eight years, with wound isolates accounting for $24 \%$ of total A. baumannii specimens. Moreover, the percentage of MDR A. baumannii isolates recovered was higher among combat casualties deployed overseas (52\%) than among local patients (20\%) [46]. Among military patients from a tertiary care burn center, patients with $A$. baumannii infection had more severe burns and comorbidities, longer lengths of stay, and higher mortality compared to patients without infection [47]. Therefore, and specifically to military personnel with war wounds, A. baumannii represents a challenging threat, and should be prevented by combating nonhealing wounds that are likely to develop wound biofilms (described shortly) with prominent antimicrobial resistance [48]. In one study about Gram-negative osteomyelitis in Brazil, A. baumannii caused $21 \%$ of cases, and $40 \%$ of the isolates were carbapenem resistant [49]. Furthermore, in China, and after the Wenchuan earthquake, about $14 \%$ of wound infections in trauma patients were caused by A. baumannii [50]. 


\subsection{Urinary Tract Infections}

Although research on A. baumannii infections has primarily focused on pneumonia and bloodstream infections, recent claims are that one in five A. baumannii strains are isolated from urinary sites [51]. A. baumannii occasionally causes urinary tract infections (UTIs), especially with indwelling urinary catheters [4], and was responsible for $1.6 \%$ of ICU-acquired UTIs in one study [52]. Balfousias and colleagues reported a PDR strain of A. baumannii causing urinary tract infection in a 72-year old patient admitted to a trauma ward of a Greek hospital for femur fracture, and requiring a combination therapy of rifampicin, tigecycline, and vancomycin in their maximum doses to produce negative urine cultures [53]. In a study of characteristics of A. baumannii isolated from ICU in 10 hospitals in Korea, $55.6 \%$ of the isolates were associated with urinary tract infection. Of these isolates, $19.8 \%$ were resistant to imipenem and $25 \%$ to meropenem, $13.5 \%$ to polymyxin B, and $17.7 \%$ to colistin [54]. Moreover, it is unusual for this organism to cause uncomplicated UTI in healthy outpatients [2].

\subsection{Meningitis}

Nosocomial meningitis due to A. baumannii remains an increasing threat in intensive care neurosurgery units, with mortality approaching $70 \%$, especially in patients on indwelling ventriculostomy tubes or cerebrospinal fistulae and receiving post-surgical antimicrobial therapy [55]. The largest case series of postneurosurgical A. baumannii meningitis published in 2019 revealed that $21 \%$ of isolates had XDR phenotype with sensitivity to only colistin and tigecycline. Furthermore, age over 40, presence of external ventricular drain, raised cerebrospinal fluid white blood cell count, and presence of comorbidities (diabetes and hypertension) were risk factors for mortality due to A. baumannii in the neurosurgical ICU [56]. In a review of pediatric meningitis cases caused by A. baumannii in China, cultures of cerebrospinal fluid yielded MDR, XDR, and PDR A. baumannii following neurosurgery, with high mortality rate [57]. In another report from China, and in a university hospital, the prevalence of MDR and XDR A. baumannii infection among patients with intracranial infection after a neurosurgical operation was $33.64 \%$. The isolates were $100 \%$ resistant to imipenem and meropenem, $98.38 \%$ to cefazolin, $100 \%$ to ceftazidime, $100 \%$ to cefatriaxone, and $98.39 \%$ to cefepime, but completely sensitive to polymyxin B, $60.66 \%$ to tigecycline, and $49.18 \%$ to amikacin. No significant differences in basic clinical data were observed between the two groups [58].

\section{A. baumannii Virulence Factors}

In recent years, approaches involving genomic, phenotypic, and infection model analyses helped in the identification of virulence factors important for A. baumannii pathogenicity [22]. Current consensus supports a multifactorial and combinatorial strategy, with about 16 identified gene islands implicated in virulence, signifying that the organism devotes a considerable portion of its genes to pathogenesis [59]. Systematic studies failed to identify a particular virulence factor responsible for clinical success of A. baumannii. This perspective highlights strong adaptive potential and could be a result of adaptation to different human body sites or pathogenic strategies, as known for other bacteria like Escherichia coli and Legionella [60]. An illustration of virulence determinants identified in A. baumannii so far is discussed below and is curtailed in Figure 2. 


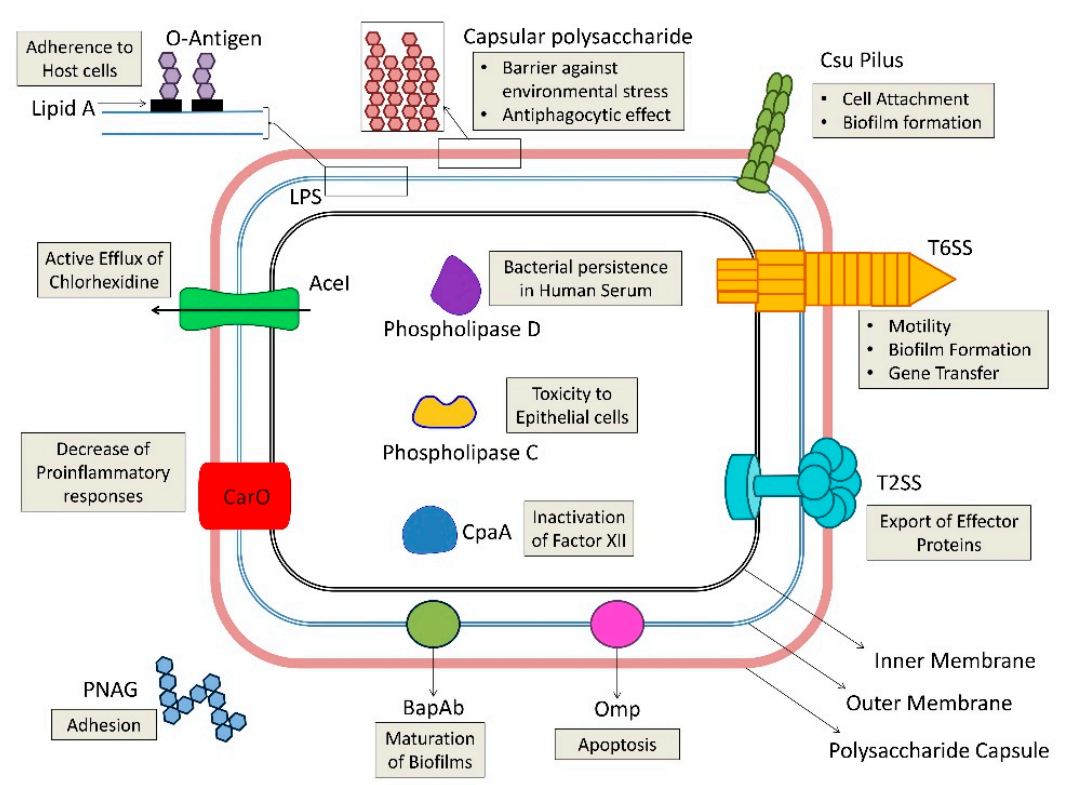

Figure 2. An illustration of virulence determinants possessed by Acinetobacter baumannii. The function of each determinant is shown in the adjacent box. AceI = Acinetobacter chlorhexidine efflux protein; $\mathrm{CpaA}=$ glycan-specific adamalysin-like protease; $\mathrm{Csu}=$ chaperon/usher pilus system; LPS = lipopolysaccharide; Omp = outer membrane protein; PNAG = poly- $\beta-1,6-\mathrm{N}$-acetylglucosamine; T2SS = type II secretion system; T6SS = type VI secretion system.

\subsection{Outer Membrane Proteins (Porins)}

Outer membrane proteins of Gram-negative bacteria generally have a pivotal role in environmental interaction and adaptation, and are key players in virulence [61]. The main outer membrane protein OmpA of A. baumannii is involved in cell invasion and apoptosis. This $38 \mathrm{kDa}$ protein is vital for small solute penetration. It binds to host cell surface, gets localized in both mitochondria and nuclei, and induces cell death [62]. In a study investigating potential of A. baumannii to invade epithelial cells of a murine pneumonia model, prominent lung histopathologic changes (abundance of white blood cells and alveolar damage) were detected in mice having wild-type infection but not in those infected with OmpA- mutants [63]. Besides its transport function as porin, OmpA has the capacity of inducing host cell apoptosis, biofilm formation, dissemination into bloodstream, and interaction with epithelial cells mainly using host fibronectin [64].

Another outer membrane protein of A. baumannii is the Omp 33- to 36-kDa protein that acts as a channel for water and whose expression is associated with resistance to carbapenem antibiotics [65]. In one study, this protein was released in immune and connective cells, where it induced apoptosis by blockade of autophagy, enabling intracellular persistence with subsequent development of cytotoxicity [66]. Furthermore, knockout strains of A. baumannii with deficient Omp 33-36 had defective growth rate and significantly reduced capability of adherence, invasion, and cytotoxicity, indicating that Omp33-36 plays an important role for fitness and virulence of $A$. baumannii [65].

Like Omp 33-36, CarO plays also a role in carbapenem resistance in A. baumannii; it was shown that increased CarO expression delays infiltration of pulmonary neutrophils via attenuation of proinflammatory responses in the trachea and lungs, allowing bacterial proliferation and resulting in severe pneumonia [67].

\subsection{Cell Envelope Factors (LPS and Capsule)}

In Gram-negative human pathogens, the cell wall polysaccharide (LPS) is one of the virulence factors involved in multiple steps of disease process. A. baumannii LPS is important for resistance to normal human serum and confers a competitive advantage for survival in vivo. It also can elicit a 
proinflammatory response in animal models [68]. The antigenic O-polysaccharide of the LPS, together with pili, might promote adherence to host cells as a first step of colonization [69].

Beyond the LPS, a major cell structure determinant of A. baumannii virulence is the presence of a capsule around bacterial surface. The repetitive, closely packed sugar units of the capsule create a barrier against environmental conditions like dryness and disinfection, and immune system reactions like phagocytosis; it also protects against some antimicrobials [70,71]. Despite differences in capsular sugars of A. baumannii, with over 100 variable types, the capsule is perpetually effective for survival of the pathogen during infections and its ability to grow in serum [72].

\subsection{Enzymes}

Phospholipases are known additional virulence factors of $A$. baumannii; these are crucial hydrolytic enzymes and possess a lipolytic activity against phospholipids of human cell membranes. While the enzyme phospholipase D helps A. baumannii to persist in human serum as shown in a murine pneumonia model, another enzyme, phospholipase $C$, is toxic to epithelial cells [73].

Data about enzymes in A. baumannii continue to culminate; recently, the enzyme CpaA, a glycan-specific adamalysin-like protease, was identified as a virulence factor that inhibits blood coagulation through inactivation of factor XII. As such, CpaA attenuates formation of thrombi in intravascular sites, promoting dissemination capacity of $A$. baumannii [74].

\subsection{Capsular Polysaccharide Composition and Outer Membrane Resistance to Desiccation and Disinfection}

Desiccation resistance, or persistence in dry environments, can allow A. baumannii strains to survive up to 100 days, although this period is variable [75]. A. baumannii environmental survival is related to presence of capsular polysaccharides surrounding the whole cell and providing defense against the environment [76]. Resistance to dryness has been linked to outer membrane composition; a mutant strain of A. baumannii with chemically altered lipo-oligosaccharide was unstable in dryness, suggesting that fluidity of the outer membrane resulting from changes in its lipid structure allows escape of water and nutrients to outside of the cell [77].

A. baumannii is shown to actively pump chlorhexidine, an antiseptic used against a wide range of bacteria by disrupting cell membranes. The Acinetobacter chlorhexidine efflux protein (AceI) is responsible for such pumping, possibly promoting survival under stressful conditions [78]. On the other hand, ethanol promotes growth and virulence of A. baumannii [79]. Ethanol in the serum of alcoholic subjects weakens phagocytosis and hence destruction of the organism [80]. Likewise, excess human consumption of ethanol is a probable predisposing factor for A. baumannii community-acquired infections [35].

\subsection{Biofilm Production and Quorum Sensing}

Among all virulence determinants, biofilm formation has become a major pathogenesis feature for A. baumannii, making the organism multidrug resistant. By definition, biofilms are microcommunities of microbes enclosed in an extracellular substance, and rendering microbes resistant to stresses including desiccation, immune system clearance, and antibiotics [81]. Biofilms additionally mediate pathogen-host interactions in A. baumannii. Most A. baumannii have a chaperon/usher pilus system, known as Csu pili, regulated by the BfmRS two-component (TC) system, a network of molecules that influences gene expression and enables building a protective capsule in response to antibiotics. The system also mediates pili formation to facilitate cell attachment [82]. Another TC system, GacSA, can affect Csu gene expression, and therefore may have an indirect influence on the pathogen's ability to produce biofilms [83]. A. baumannii is 'characterized by production of biofilm-associated proteins (BapAb) similar to Bap protein of Staphylococcus aureus, and that have capacity to aggregate for building up biofilm matrix in response to stressful environmental conditions [84].

Although most sequenced strains of $A$. baumannii carry a bap gene, many seem to have disrupted or truncated bap sequences. Nevertheless, Bap-like proteins, BLP1 and BLP2, may be 
encoded by some strains of A. baumannii, and help in maturation of biofilms, like BapAb [85]. Another notable factor that helps $A$. baumannii to produce biofilm is production of the exopolysaccharide poly- $\beta-1,6-N$-acetylglucosamine (PNAG), which is produced by many Gram-negative species, and is essential for adhesion and aggregation [86].

Many reports suggest that quorum sensing plays a major role in biofilm formation. Quorum sensing is a mode of communication among bacteria to maintain population density, usually by production of signaling molecules known as autoinducers [87]. These are hormone-like compounds, including acyl homoserine lactones (AHLs), that are responsible to regulate motility, biofilm formation, and other characteristics. The quorum sensing cycle in A. baumannii includes AbaI inducer as well as its cognate receptor $\mathrm{AbaR}$. AbaI, encoded by the gene $a b a I$, is a sensor protein that functions as an autoinducer synthase producing signal AHL molecules, while AbaR functions as a receptor protein, which upon binding to AHLs induces a cascade of reactions. This binding triggers production of more AHLs in a positive feedback loop manner, which results in regulation of biofilm formation [88].

\subsection{Motility}

Bacterial motility contributes to the infectious ability and increased virulence of some bacteria [89]. A. baumannii lacks flagella and has been long labeled as non-motile [90,91]. However, studies show that this organism can survive during infection and can spread on surfaces during hospital survival by using twitching motility [92]. Such motility consists of extension and retraction movements to push cells in media, by means of type IV pili [93]. In addition to motility, these pili are also responsible for biofilm formation and gene transfer [94]. In A. baumannii, a model of Caenorhabditis elegans infection showed higher virulence due to motility [95]. Investigations comparing blood isolates of A. baumannii to sputum isolates found that the former isolates were more motile, probably indicating a higher survival advantage in blood [96].

In addition to twitching motility, some isolates of $A$. baumannii move on living and non-living surfaces without relying on flagella by another mode of motility called surface-associated motility [97]. This type of motility also requires type IV pili, quorum sensing, lipo-oligosaccahride production, and 1,3-diaminopropane, which mediates signaling needed for impacting surface-associated motility by quorum sensing [90].

\subsection{Micronutrient Acquisition Systems}

A major factor contributing to A. baumannii persistence as a nosocomial pathogen is its ability to capture host nutrients, including iron, manganese, and zinc, thus adapting to metal-limited environment imposed by the host [98]. The main mechanism used by A. baumannii for capturing iron involves five clusters of high-affinity iron-chelating molecules known as siderophores. In addition, the organism possesses transporters and receptors for direct iron uptake such as FecA and FecI, which allow the utilization of heme [99]. Models of iron transporter damage have shown to reduce virulence by decreasing biofilm production and resistance to oxidative stress [100].

Moreover, A. baumannii relies on a high-capacity zinc scavenging system, consisting of ZnuABC transporter and ZigA GTPase; ZnuABC assures intracellular zinc uptake, while ZigA is responsible for zinc metabolism [101,102]. As such, A. baumannii circumvents calprotectin, an immune system protein, that innately complexes zinc, manganese, and divalent metal ions, thus inhibiting bacterial growth. In a murine pneumonia model, zig $A$ mutants with depleted zinc availability had less systemic dissemination from the lungs following infection [102]. Although mechanisms employed by A. baumannii to overcome manganese limitation are less understood, it is postulated that a transporter belonging to the family of resistance-associated macrophage protein (NRAMP) facilitates manganese accumulation and growth in presence of calprotectin [103]. 


\subsection{Protein Secretion Systems}

In A. baumannii, secretion of proteins from cell surface structures allows the pathogen's interaction with environment and hosts, and hence represents attractive targets for treatment $[104,105]$. The trimeric autotransporter (Ata) was the first secretion system described in A. baumannii. Ata mediates attachment to human matrix components, particularly collagen, and it is also implicated in biofilm formation/maintenance and virulence [104]. A. baumannii also uses a type II secretion system (T2SS) to export multiple effector proteins. In this two-step secretion process, the general secretory pathway (Sec) or the Twin-arginine (Tat) system deliver proteins with an N-terminal secretion signal across the pathogen's inner membrane. Next, the secretion signal is removed, and T2SS machinery secretes folded proteins to outside of the cell [106]. T2SS effectors include CpaA, LipA, and LipH, where LipA and LipH are lipases that are essential for utilization of exogeneous lipids, and CpaA is a metallo-endopeptidase that degrades fibrinogen and factor $\mathrm{V}$ in a zinc-dependent mechanism, negatively influencing blood clotting pathways [107].

Like many Gram-negative bacteria, A. baumannii encodes type VI secretion system (T6SS), a multi-component secretion machine capable of injecting protein toxins into other bacteria in a contact-dependent manner, thus making it important in polymicrobial infections [108]. A single, conserved, genetic locus encodes 13 core structural proteins of this system, while additional, accessory, or regulatory elements may co-exist. Assembled T6SS system contracts to allow release of the effector protein, which typically targets other bacteria without self-intoxication [104]. In A. baumannii, T6SS may attack other bacteria, where it produces toxins like nucleases, peptidoglycan hydrolases, or cell-membrane active toxins [109]. Interestingly, clinical isolates with active T6SS are isolated at higher frequencies from immunocompromised patients, suggestive of competitive advantage against concomitant pathogens [110].

\subsection{Others}

Although tangible progress was accomplished in A. baumannii pathogenesis studies, emerging areas are still under in-depth investigation. Among these, outer membrane vesicles, long recognized to protect bacteria against host innate immunity, are being studied for their ability to provide mechanisms for secretion of virulence factors in A. baumannii [99]. Possibilities also exist that A. baumannii may acquire a toxin-like virulence factor like other human pathogens such as Vibrio cholerae [111]. Indeed, ongoing research on molecular mechanisms utilized by this pathogen for survival within the host and adaptation to environmental stress could uncover additional important pathogenesis features.

\section{Mechanisms of Antibiotic Resistance in A. baumannii}

The increased antimicrobial resistance in A. baumannii and the occurrence of strains resistant to virtually all available drugs is quite alarming [4]. Intrinsically resistant to a number of commonly used antibiotics, such as aminopenicillins, first- and second-generation cephalosporins, A. baumannii has a quite notable ability to acquire resistance to numerous other agents and thus swiftly respond to changes in environmental pressure [2]. The current guidelines of treatment of infections by A. baumannii are seriously affected by such resistance inclination, rendering options of chemotherapy rather limited [36]. For susceptible organisms, first-line therapy consists of a carbapenem, such as imipenem-cilastatin, meropenem, or doripenem [112], where imipenem was historically considered the gold standard for management of VAP caused by this organism [113]. The clinical cure rates with imipenem for A. baumannii-induced VAP range from $57 \%$ to $83 \%$. Because isolates susceptible to imipenem may be resistant to meropenem and vice versa, it is advisable to test susceptibility to the specific carbapenem prior to its clinical use [114]. Nevertheless, as explained shortly, carbapenem resistance rates for A. baumannii have been rising dramatically worldwide, rendering the antibiotic armamentarium more restricted, and clinical practice is shifted towards alternative agents [36]. Of these, colistin (polymyxin E) and polymyxin B are being used to treat A. baumannii VAP, bacteremia and meningitis [113]. A major 
limitation to colistin is high rates of nephrotoxicity and neurotoxicity, as well as poor penetration into the lung tissue, incumbering its utility [115]. Another alternative agent is minocycline, where successful clinical and microbiologic outcomes were reported for patients with $A$. baumannii VAP as well as skin and soft tissue infections [116]. Tigecycline, an alternative antibiotic for MRD and XDR strains of A. baumannii, has been used with variable success rates $[117,118]$. Despite reasonable in vitro activity of this agent against $A$. baumannii, clinical data remain limited. Specifically, in clinical trials with A. bauamnnii VAP and bacteremia, patient outcomes with tigecycline have been inferior to other agents [119]. An interesting alternative to both tetracyclines, minocycline and tigecycline, may be the $\beta$-lactamase inhibitor, sulbactam, which has direct antimicrobial activity against $A$. bauamnnii, via its intrinsic affinity for A. baumannii penicillin-binding proteins [36]. A major disadvantage of sulbactam is that it is only available in combination with ampicillin in the United States, besides emerging resistance, warranting further investigations into the clinical utility of this agent [120].

Combination therapy is frequently used in A. baumannii infections as a strategy to increase antibiotic coverage before drug susceptibility testing results are known, to decrease the risk of resistance, and to improve patient outcomes. However, there are no definitive clinical data to support its use for these purposes, and results from human trials are limited [112]. For example, therapy with polymyxin B plus another agent (imipenem, meropenem, rifampin, ampicillin-sulbactam, or others) was associated with a lower mortality rate than with polymyxin B monotherapy [121]. A randomized, multicenter trial done on patients with XDR A. baumannii infections treated with colistin and rifampin versus colistin alone, showed superior microbial eradication in patients randomized to the combination arm, but no difference in mortality [122]. Other combination regimens suggest increased efficacy of sulbactam with cefepime, meropenem, imipenem, amikacin, or rifampin [123]. Alternative combinations include colistin-tigecycline and colistin-carbapenem therapy, with the latter perhaps the most supported [36], and have been recommended in several trials [124-126]. Furthermore, a recent investigation has highlighted a remarkable synergistic interaction between colistin and vancomycin [127].

A clear consensus regarding the most appropriate combination therapy for resistant $A$. baumannii infections remains to be established, pending more comprehensive clinical studies. Until these become accessible, and until novel antimicrobial entities, bacteriophages, or antimicrobial peptides, which are under thorough scientific investigation, become established, resistance of A. bauamnnii to contemporary therapies will escalate. Figure 3 and Table 1 summarize the antibiotic resistance mechanisms in A. baumannii; an elaboration of these mechanisms with literature citing them is presented below.

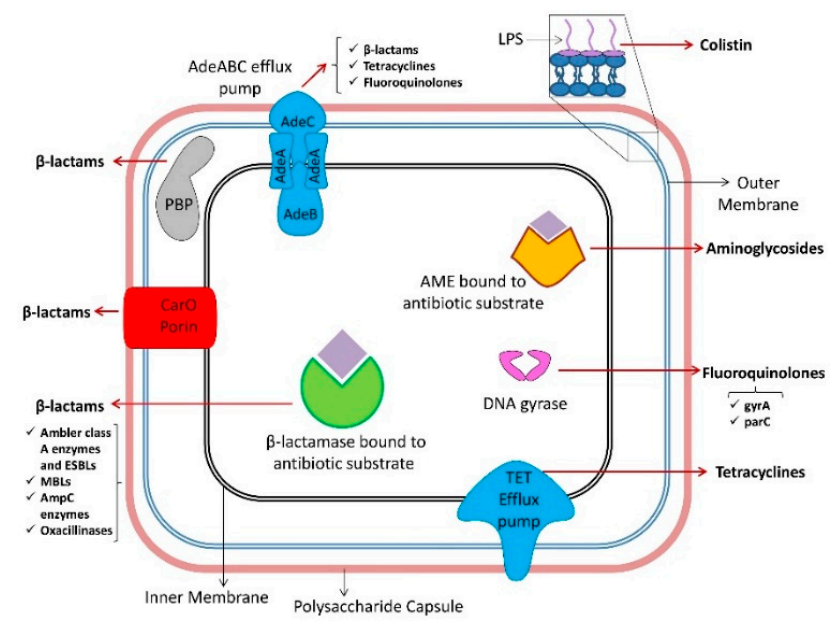

Figure 3. Diagram of various resistance mechanisms of Acinetobacter baumannii to antimicrobial agents. Antibiotic modifying enzymes, efflux pumps, porins, drug targets, and the affected antibiotics by each resistance mechanism are shown. AMEs = Aminoglycoside modifying enzymes; AmpC = Ambler class $C$ cephalosporinases; ESBLs = Extended-spectrum $\beta$-lactamases; MBLs = Metallo- $\beta$-lactamases; LPS = Lipopolysaccharide; PBP = Penicillin binding protein . 


\section{1. $\beta$-lactams}

The most prevalent mechanism of resistance of $A$. baumannii to $\beta$-lactams is enzymatic hydrolysis by $\beta$-lactamases; all of the four Ambler classes of these enzymes have been described in this organism, in addition to nonenzymatic pathways.

\subsubsection{Ambler Class A Enzymes}

Class A $\beta$-lactamases are serine-dependent enzymes inhibited by clavulanate or tazobactam. They hydrolyze all penicillins and cephalosporins with the exception of cephamycins [128]. CTX-M, GES, PER, SCO, SHV, TEM, and VEB are among identified class A $\beta$-lactamases in A. baumannii [129-132]. While some of these, like SCO-1 and TEM-1, are narrow-spectrum, others like CARB-10, CTX-M-2, CTX-M-15, GES-14, PER-1, PER-7, and SHV-5 are extended spectrum $\beta$-lactamases (ESBLs) [133,134]. Some GES enzymes with carbapenem-hydrolyzing activity, such as GES-11, have been detected in A. baumannii $[135,136]$.

In 2010, Robledo et al. reported, for the first time, Ambler class A Klebsiella pneumoniae carbapenemase (KPC)-5 in MDR A. baumannii from Puerto Rico [137]. Additional reports described KPC-3 in A. baumannii a Portugese University hospital [138], and both KPC-2 and KPC-3 in isolates from Brazil [139]. In a recent meta-analysis about $A$. baumannii in burn injury patients, the prevalence of KPC was over 16\% [140]. Carriers of a KPC variants are usually MDR microorganism, and standard medical treatment becomes ineffective with high mortality rates [139]. The true prevalence of KPC and its probable association with other resistance determinants should be investigated carefully, especially with reports of already globally disseminated A. baumannii ST2 that carries bla $a_{\mathrm{KPC}}[141]$.

\subsubsection{Ambler Class B Enzymes}

These are zinc-dependent metallo- $\beta$-lactamases (MBLs), that strongly hydrolyze all $\beta$-lactams, including carbapenems, but not aztreonam [142], and are inhibited by metal chelators like EDTA and dipicolinic acid [128]. The most concerning among MBLs is NDM-1, with carbapenem-resistant A. baumannii producing NDM-1 being identified in 2011 in China [143]. Additional reports of NDM-1 in A. baumannii have accumulated from Tunisia [144], Iran [145], Lebanon [146], and Saudi Arabia [147]. Apart from NDM, VIM [148], GIM [149], SIM [150], and IMP [151,152], including a new allelic variant, IMP-55 [153] were reported.

The majority of acquired MBL genes in A. baumannii lie on class 1 integrons, which simultaneously harbor other resistance genes, like aminoglycoside resistance [154]. Such strains are significantly more resistant than strains without integrons, and, clinically, this implies that use of one antibiotic can result in overexpressed resistance to other antibiotics, especially with genetic location of integrons on mobile elements like plasmids or transposons, making them easily transferrable [155].

\subsubsection{Ambler Class C Enzymes}

Also known as Acinetobacter-derived cephalosporinases (ADCs), chromosomally encoded AmpC cephalosporinases are intrinsic to all A. baumannii strains [155]. They mediate cephamycin (cefoxitin and cefotetan), cephalosporin, penicillin, and $\beta$-lactamase inhibitor combinations resistance, but are not affected by $\beta$-lactamase inhibitors like clavulanate and sulbactam [156]. Unlike other Gram-negative bacteria, A. baumannii does not induce expression of AmpCs. Overexpression is usually mediated by inserting ISAba1 before AmpC genes, enhancing $A$. baumannii resistance to extended-spectrum cephalosporins [157]. Cefepime and carbapenems appear to be stable in the presence of these enzymes [155]. Whole-genome sequencing approaches are allowing detection of new AmpC alleles like AmpC-69, AmpC-70, and AmpC-71 [158]; the new AmpC allelic variant encoded by bla ADC-196 was also recently identified in a clinical A. baumannii isolate from China [159]. 


\subsubsection{Ambler Class D Enzymes}

$\beta$-lactamases of class $D$, or the oxacillinases (OXAs), are serine-dependent and commonly hydrolyze oxacillin much faster than benzylpenicillin, hence the name [156]. In various bacteria, over 400 OXA-type enzymes are already known, of which many are carbapenemases [133]. A. baumannii isolates harboring plasmid-encoded OXA-23, OXA-24/40, OXA-58, OXA-143, and OXA-235 families have appeared from the 1980s onwards; chromosomal loci encoding some of these enzymes were also identified [20,160-163].

The first isolation of a carbapenem-hydrolyzing class D oxacillinase from A. baumannii dates back to 1985, where it was isolated from blood culture of a Scottish patient, and termed ARI-1 [164], which is now referred to as OXA-23 [165]. This enzyme is currently disseminated worldwide [166], and the insertion sequence, ISAba1, in the $b l a_{\mathrm{OXA}-23}$ promoter is associated with overexpression [21]. A. baumannii naturally produces chromosomally encoded OXA-51-group carbapenemase at a low level, and acquisition of a strong promoter by ISAba1, analogous to the case with ADCs, upstream of the OXA-51-group gene may lead to elevation of carbapenem MICs [167].

It is worth mentioning that OXA-23 enzymes have been identified in many areas of the world in concurrence with other carbapenemases in A. baumannii clinical isolates. For instance, OXA-23 co-exists with GES-11 in reports from Lebanon [136] and Kuwait [168], with NDM-1 in reports from India [169], and with OXA-58 in reports from Tunisia [170]. In a study from Thailand, a rare, worrisome case of A. baumannii exhibited OXA-23, VIM-2, and NDM-1 [171], highlighting the versatility of carbapenemases that can be harbored simultaneously by this organism.

\subsubsection{Nonenzymatic $\beta$-lactam Resistance Mechanisms}

Apart from $\beta$-lactamases, $\beta$-lactam resistance in A. baumannii is additionally ascribed to nonenzymatic mechanisms, perhaps less well elucidated than enzymatic hydrolysis, but mainly including changes in outer membrane proteins and multidrug efflux pumps [155]. The loss of the 29-kDa outer membrane porin, $\mathrm{CarO}$, was associated with imipenem and meropenem resistance [172]. A recent study from Egypt revealed that ISAba1 has been inserted in carO gene leading to interruption of its expression [173]. A report about an insertion sequence disrupting the gene encoding the penicillin binding protein PBP6b (also known as dacD) was identified in an endemic carbapenem-resistant clone in a Spanish hospital [174]. Additionally, a collective set of changes involving disruptions in carO and $d a c D$, as well as carbapenemase production, developed resistance to carbapenems among clinical isolates of A. baumannii [175]. The role of other porins like OmpA, Omp33, OprB, Omp25, OprC, OprD, and OmpW is also reported [176].

Multi-drug efflux systems play a major role in $\beta$-lactam resistance in $A$. baumannii. The resistance-nodulation-division (RND) family-type pump AdeABC is the best studied and has a broad substrate range including $\beta$-lactams, aminoglycosides, erythromycin, chloramphenicol, tetracyclines, fluoroquinolones, and trimethoprim [155]. AdeABC has a three-component structure: Outer membrane protein (adeC), multidrug transporter (adeB), and membrane fusion protein (adeA) [177]. This pump is chromosomally encoded and regulated by a TC system with a sensor kinase (AdeS) and an associated response regulator (AdeR) [178]. Either point mutations or insertion of ISAba1 sequence in adeS gene leads to overexpression of AdeABC [133]. In a Chinese report, high expression of AdeABC in $A$. baumannii was closely associated with meropenem resistance. The upregulation of ade $A$ and adeB expression was not due to gene mutations in the regulatory genesadeS and adeR, suggesting possible involvement of other pathways for efflux pump overexpression [179].

\subsection{Tetracyclines and Glycylcyclines}

Similar to other Gram-negative organisms, tetracycline resistance in A. baumannii occurs primarily via energy-dependent efflux pumps, with a lesser extent of resistance attributed to ribosomal protection mechanisms through encoding proteins that shield bacterial ribosomes [180]. Tetracycline efflux 
pumps of A. baumannii fall into two categories: RND pumps, which are nonspecific, constitutive pumps, and Tet efflux pumps with TetA conferring resistance to tetracycline but not minocycline or doxycycline and TetB resulting in resistance to minocycline and tetracycline, tigecycline remaining unaffected [181]. The general structure of RND pumps includes three parts, namely, AdeA, AdeB, and $A d e C$ in $A$. baumannii. These respectively encode membrane fusion, multidrug transporter, and outer membrane pump elements [178]. Gene disruption analysis proved that RND pumps can eject tetracyclines, elevating MICs for tigecyline, minocycline, and tetracycline [182]. Other RND pumps like AdeIJK exist but may play a less profound role on tetracycline efflux and can be synergistic with AdeABC [183].

According to studies from many countries, overexpression of the efflux pumps AdeABC and AcrAB-TolC efflux systems was observed in clinical tigecycline-resistant isolates [184-186]; this is increasingly affecting utility of this modified tetracycline in treatment. Tigecycline has broader spectrum of activity compared to earlier tetracyclines, has good tissue penetration, and is stable against many tetracycline resistance mechanisms including Tet efflux pumps, as well as against ribosomal protection, such as Tet(O) and Tet(M) [187]. Control of tigecycline usage should be considered to reduce emergence of resistance.

Resistance to minocycline in A. baumannii is rare and has been attributed to tet $M$, a ribosomal protection gene [181]. This gene possesses almost 100\% homology to $S$. aureus gene, and may represent transfer of resistance mechanisms between the two pathogens [188]. Because tet $M$ encodes a GTPase analogous to the elongation factors EF-G and EF-Tu, it can mediate tetracycline release from bacterial ribosome by a GTP-dependent mechanism, through competition with EF-G for an overlapping binding site. By dissociation of tetracycline from its ribosomal binding site, tet $M$ enables translation to continue in the presence of tetracycline [189]. Recent evidence shows that tetM is encoded by A. baumannii isolated from wastewater treatment plants [190] and animal culture ponds [191], indicating extensive dissemination; the assessment of such resistance pools is critical not only for clinical significance, but also for environmental protection.

\subsection{Fluoroquinolones}

The emergence of resistance to fluoroquinolones in A. baumannii results from mutations of the fluoroquinolone target enzymes, DNA gyrase and DNA topoisomerase IV, respectively encoded by the genes gyrA and parC. [192]. These mutations mainly affect the fluoroquinolone-resistance determining regions (QRDRs) of the target enzymes, with common amino acid substitutions being Ser 83 and Gly 81 within gyrA, and Ser 80 and Glu 84 within parC. [193]. Such mutations decrease affinity of fluoroquinolones to the enzyme-DNA complex. Clinically significant resistance to fluoroquinolones may be achieved with only a single mutation in gyr $A$; however, double amino acid of both gyrA and parC produce augmented resistance compared to single ones [194]. There has not been evidence of mutations in $\operatorname{par} C$ without a concurrent mutation in $g y r A$, which suggests that DNA topisomerase IV could be a complementary target for fluoroquinolones [193]. So far, plasmid-mediated fluoroquinolones resistance genes like $q n r A, q n r B$, and $q n r S$ have not been identified in epidemiologic studies of $A$. baumannii.

Moreover, moderate level fluoroquinolone resistance in $A$. baumannii may result from chromosomal efflux pumps [81]. Efflux pump inhibitors can reverse multidrug resistant phenotype of A. baumannii [194]. Mutations of a two-step regulator (AdeR) and sensor (AdeS) of the previously mentioned AdeABC efflux pump belonging to RND family of pumps resulted in higher fluoroquinolone efflux [192]. Finally, quinolones are the principle substrates of the efflux pump AbeM, resulting in clinically significant MIC changes for ciprofloxacin and norfloxacin [195]. It is worth mentioning that AbeM is a hydrogen-ion-coupled member of the multidrug and toxic compound extrusion (MATE) family. 


\subsection{Aminoglycosides}

Aminoglycoside resistance in A. baumannii results from production of aminoglycoside-modifying enzymes (AMEs), which can be categorized into various groups with different chemical actions, including acetyltransferases, adenyltransferases, and phosphotransferases [196]. Such AMEs alter corresponding functional groups on aminoglycosides, weakening the binding capacity of these antibiotics at their ribosomal target sites. AMEs are often found within class 1 integrons and can be located on either plasmids or chromosomes [197]. The action of AMEs is selective, whereby they differently affect various aminoglycoside molecules. For instance, A. baumannii phosphotransferases can make many aminoglycosides, including amikacin, inactive. Gentamicin and tobramycin retain activity because of their inability to accept phosphate secondary to a lack of $3^{\prime}$-hydroxyl groups [198]. A. baumannii isolates may produce a combination of AMEs, as in a PDR A. baumannii strain described in China and carrying four AMEs [199]. Among others, AAC(3)-Ia, ANT(2')-Ia, and ANT(3")-Ia are variants of AMEs described in A. baumannii [186,200,201].

Another resistance mechanism to aminoglycosides is the production of $16 \mathrm{~S}$ rRNA methylase genes $\operatorname{arm} A, r m t A, r m t B, r m t C$, and $r m t D$, which alter target binding site for aminoglycosides within the $30 \mathrm{~S}$ ribosomal subunit. Unlike AMEs, methylases induce high-level resistance across all clinically useful aminoglycosides, including gentamicin, tobramycin, and amikacin [198]. The armA gene is found among other Gram-negative organisms, is plasmid-borne, and lies within a transposon (Tn1548) [202].

Although AMEs remain the principle aminoglycoside resistance mechanism in A. baumannii, these antibiotics are also subject to efflux pump ejection outside the cell. Gentamicin is subject to effective efflux by AdeABC and AbeM pumps, while these two pumps are less efficient in extruding the more hydrophilic aminoglycosides, amikacin and kanamycin [176,203].

\subsection{Macrolides}

While azithromycin shows variable activity against some $A$. baumannii isolates, this does not really appear significant for clarithromycin and erythromycin [203]. There are only scarce reports in literature on successful treatment of infections caused by A. baumannii with macrolides; however, A. baumannii with a mutant AbeS small multidrug resistance (SMR) pump exhibits erythromycin and chloramphenicol resistance [204]. Recently, an investigation from Japan [205] proved that the MacA-MacB-TolC tripartite complex transmembrane machine of A. baumannii that spans both the inner and outer membranes exists as a unique type of transporter. Macrolides, virulence determinants, peptides, and cell envelop elements appear to be important substrates of this transporter.

\subsection{Polymyxins}

Polymyxin E, also known as colistin, is an old antibiotic belonging to the polymyxin family, first introduced in the 1950s; owing to its harmful effects on renal function, its use was banned by many countries [206]. Nevertheless, the rapid emergence of resistance in A. baumannii to multiple antibiotics, including carbapenems, has revived interest in the use of colistin. Currently, resistance to this latter antibiotic in A. bauamannii is on the rise [207].

Primary colistin resistance mechanisms in A. baumannii are chromosomally encoded, and involve (i) phosphoethanolamine (PetN) addition to lipid A of the outer membrane altering its structure, (ii) mutation of genes needed for lipid A synthesis leading to its complete loss, (iii) of outer membrane low expression of proteins needed for outer membrane stability, and (iv) deficient expression of LPS synthesis cofactors [207]. Polymyxins are cationic amphipathic compounds, and initially interact with the negatively charged lipid A component of LPS. Controlled addition of positively charged residues such as PetNt to LPS reduces negative charge on bacterial surface and therefore limits interaction between the polymyxin and the LPS [208]. The expression of PetN transferases is regulated by the concerted action of TS systems [209]. Colistin resistance in A. baumannii clinical isolates is associated with alterations in the $p m r C A B$ operon. The $p m r C$ gene codes for a PetN transferase, and $p m r A$ and 
pmrB code for TS system. Mutations in the PmrAB TS system induce overexpression of $p m r C$, leading to modification of lipid A with PetN and colistin resistance [210]. Uniquely, A. baumannii strains can also become highly resistant to polymyxins via spontaneous mutations in lipid A biosynthesis such that they produce no LPS or lipid A. If the biosynthetic lipid A genes, $l p x A, l p x C$, or $l p x D$, become completely inactive, LPS is lost, elevating colistin MIC due to lack of LPS interaction with this antibiotic [211].

Recently, additional genes (lpsB, lptD, and vacJ) were shown to contribute to polymyxins resistance in A. baumannii. These genes reduce fluidity and increase osmotic resistance of the outer membrane. In overexpression mutations of these genes, polymyxin resistance in A. baumannii occurs [212]. The levels of biotin are essential for susceptibility to polymyxins in A. baumannii. Biotin is a co-factor of lipid metabolism, involved in a rate-limiting step in fatty acid synthesis. Accordingly, high levels of biotin promote increased lipid A synthesis, with higher sensitivity to colistin. On the other hand, mutations in genes needed for biotin synthesis reduces effectiveness of colistin [212]. Hood and colleagues showed that removal of a particular locus of $l p s B$, which is involved in biotin biosynthesis can result in colistin resistance in A. baumannii [213].

Resistance to colistin in A. baumannii was originally chromosomal, which limits its rapid distribution and dissemination [207]. However, the plasmid-borne $m c r-1$ gene was identified in Escherichia coli of animal, human, and environmental origin from China in 2015 [214]. Subsequently, $m c r-1.2, m c r-2, m c r-3, m c r-4$, and $m c r-5$ variants were also identified [215]. Diverse plasmids with $m c r$ genes are nowadays described in Enterobacteriaceae from many countries. This gene encodes a PetN transferase resulting in polymyxin resistance. Despite that, $m c r$ has not been described in A. baumannii until recently in reports from Pakistan [206] and Brazil [216], probably highlighting the high tendency for spread and stressing the need to understand the actual status of global colistin resistance in this pathogen. 
Table 1. Important resistance determinants in Acinetobacter baumannii showing possible enzymes/target modifications/permeability lesions.

\begin{tabular}{|c|c|c|c|c|}
\hline Antibiotic & Resistance Mechanism & $\begin{array}{c}\text { Enzyme/target/ } \\
\text { Permeability Defect }\end{array}$ & Example & Reference \\
\hline \multirow{22}{*}{$\beta$-lactams } & \multirow{18}{*}{$\beta$-lactamases } & \multirow{6}{*}{ Ambler class A } & ESBLs of the families TEM, SHV, CTX-M, PER and VEB & [129-132] \\
\hline & & & SCO-1 (narrow spectrum) & [134] \\
\hline & & & Carbapenem-hydrolyzing ESBL of GES-type (GES-11) & {$[135,136]$} \\
\hline & & & KPC-2 & [139] \\
\hline & & & KPC-3 & {$[138,139]$} \\
\hline & & & KPC-5 & [137] \\
\hline & & \multirow{5}{*}{ Ambler class B } & NDM & [143-147] \\
\hline & & & VIM & [148] \\
\hline & & & GIM & [149] \\
\hline & & & SIM & [150] \\
\hline & & & IMP & [151-153] \\
\hline & & Ambler class $\mathrm{C}$ & AmpC-69, AmpC-70, AmpC-71, and ADC-196 & {$[2,157,158]$} \\
\hline & & \multirow{6}{*}{ Ambler class D } & OXA-23-like & {$[20,136,164,165,167,170]$} \\
\hline & & & OXA24/40-like & [159] \\
\hline & & & OXA-51-like & {$[20,166]$} \\
\hline & & & OXA-58-like & [169] \\
\hline & & & OXA-143-like & [161] \\
\hline & & & OXA-235-like & [162] \\
\hline & \multirow{2}{*}{ Permeability lesions } & \multirow{2}{*}{$\begin{array}{l}\text { Outer membrane porin } \\
\text { downregulation }\end{array}$} & CarO & {$[171,172,174]$} \\
\hline & & & OmpA, Omp33, OprB, Omp25, OprC, OprD, and OmpW & [175] \\
\hline & Efflux pump overactivity & RND pump & AdeABC & {$[2,176,177]$} \\
\hline & Target mutation & PBP & PBP6b (dacD) & {$[173,174]$} \\
\hline
\end{tabular}


Table 1. Cont

\begin{tabular}{|c|c|c|c|c|}
\hline Antibiotic & Resistance Mechanism & $\begin{array}{c}\text { Enzyme/target/ } \\
\text { Permeability Defect }\end{array}$ & Example & Reference \\
\hline \multirow{3}{*}{ Tertacyclines } & \multirow{2}{*}{ Efflux pump overactivity } & RND pump & AdeABC, AdeIJK, and AcrAB-TolC & [179-185] \\
\hline & & Tet pump & TetA and TetB & [180] \\
\hline & Ribosomal protection & Tetracycline dissociation from ribosome & Tet(O) and Tet(M) & {$[180,186-188]$} \\
\hline \multirow{4}{*}{ Fluoroquinolones } & \multirow{2}{*}{ Target mutation } & DNA gyrase & GyrA & {$[191,192]$} \\
\hline & & DNA topoisomerase IV & ParC & {$[191,192]$} \\
\hline & \multirow{2}{*}{ Efflux pump overactivity } & RND pump & AdeABC & {$[81,191]$} \\
\hline & & MATE family & AbeM & [194] \\
\hline \multirow{6}{*}{ Aminoglycosides } & \multirow{4}{*}{ Drug inactivating enzymes } & \multirow{4}{*}{ Aminoglycoside modifying enzymes } & AAC(3)-Ia & [185] \\
\hline & & & AAC(3')-Ia & [200] \\
\hline & & & ANT(2')-Ia & [200] \\
\hline & & & ANT(3")-Ia & [199] \\
\hline & Target mutation & 16sRNA methylase genes & $\operatorname{arm} A, r m t A, r m t B, r m t C$, and $r m t D$ & {$[197,201]$} \\
\hline & Efflux pump overactivity & RND pumps & AdeABC & {$[175,202]$} \\
\hline Macrolides & Efflux pump overactivity & SMR pump & AbeS & [204] \\
\hline \multirow{6}{*}{ Polymyxins } & \multirow{6}{*}{ Target mutation } & \multirow{3}{*}{ Lipid A modification by PetN transferase } & PmrC & [208-210] \\
\hline & & & MCR-1 & [214] \\
\hline & & & MCR-4 & {$[215,216]$} \\
\hline & & Lack of lipid A biosynthesis & LpxA, LpxC, or LpxD & [211] \\
\hline & & Decreased stability of outer membrane & LpsB, LptD, and VacJ & [212] \\
\hline & & Reduced biotin synthesis & LpsB & {$[207,212,213]$} \\
\hline
\end{tabular}

ESBLs = Extended-spectrum $\beta$-lactamase; PBP = Penicillin binding protein; RND = resistance-nodulation-division; MATE = multidrug and toxic compound extrusion; SMR = small multidrug resistance; $\mathrm{PetN}=$ phosphoethanolamine. 


\section{Conclusions}

A. baumannii has developed three basic properties to perfectly adapt to current healthcare settings: (i) Ability to colonize skin, mucous membranes, and devices and survive in the hospital environment; (ii) ability to express multiple virulence features; and (iii) extensive resistance to antimicrobial agents through enzymatic modification of antibiotics, target gene mutation, altered outer membrane permeability, and upregulated multidrug efflux pumps. With rapid increase in studies addressing the entire armamentarium of virulence determinants and resistance pathways possessed by this superbug, its complex influences on human health are gradually uncovered. Indeed, thorough investigations will reveal additional knowledge about this staggering pathogen, and the future holds promise for better insights into its machineries through novel research directions.

Author Contributions: D.H.H. conceptualized and wrote the first draft of this review. C.A.M. was in charge of revision and editing. All authors have read and agreed to the published version of the manuscript.

Funding: The APC was funded by Zayed University.

Conflicts of Interest: The authors declare no conflict of interest.

\section{References}

1. Clark, N.M.; Zhanel, G.G.; Lynch, J.P. Emergence of antimicrobial resistance among Acinetobacter species: A global threat. Curr. Opin. Crit. Care 2016, 22, 491-499. [CrossRef]

2. Peleg, A.Y.; Seifert, H.; Paterson, D.L. Acinetobacter baumannii: Emergence of a successful pathogen. Clin. Microbiol. Rev. 2008, 21, 538-582. [CrossRef] [PubMed]

3. Bianco, A.; Quirino, A.; Giordano, M.; Marano, V.; Rizzo, C.; Liberto, M.C.; Focà, A.; Pavia, M. Control of carbapenem-resistant Acinetobacter baumannii outbreak in an intensive care unit of a teaching hospital in Southern Italy. BMC Infect. Dis. 2016, 16, 747. [CrossRef] [PubMed]

4. Dijkshoorn, L.; Nemec, A.; Seifert, H. An increasing threat in hospitals: Multidrug-resistant Acinetobacter baumannii. Nat. Rev. Microbiol. 2007, 5, 939-951. [CrossRef] [PubMed]

5. Smiline Girija, A.S.; Priyadharsini, J.V. CLSI based antibiogram profile and the detection of MDR and XDR strains of Acinetobacter baumannii isolated from urine samples. Med. J. Islam. Repub. Iran 2019, 33, 3.

6. Assimakopoulos, S.F.; Karamouzos, V.; Lefkaditi, A.; Sklavou, C.; Kolonitsiou, F.; Christofidou, M.; Fligou, F.; Gogos, C.; Marangos, M. Triple combination therapy with high-dose ampicillin/sulbactam, high-dose tigecycline and colistin in the treatment of ventilator-associated pneumonia caused by pan-drug resistant Acinetobacter baumannii: A case series study. Infez. Med. 2019, 27, 11-16.

7. Goic-Barisic, I.; Seruga Music, M.; Kovacic, A.; Tonkic, M.; Hrenovic, J. Pan Drug-Resistant Environmental Isolate of Acinetobacter baumannii from Croatia. Microb. Drug Resist. 2017, 23, 494-496. [CrossRef]

8. WHO Publishes List of Bacteria for Which New Antibiotics Are Urgently Needed. Available online: https://www.who.int/news-room/detail/27-02-2017-who-publishes-list-of-bacteria-for-which-newantibiotics-are-urgently-needed (accessed on 21 August 2019).

9. Bouvet, P.J.; Grimont, P.A. Identification and biotyping of clinical isolates of Acinetobacter. Ann. Inst. Pasteur Microbiol. 1987, 138, 569-578. [CrossRef]

10. Vijayakumar, S.; Biswas, I.; Veeraraghavan, B. Accurate identification of clinically important Acinetobacter spp.: An update. Future Sci. OA 2019, 5, FSO395. [CrossRef]

11. Gerner-Smidt, P. Ribotyping of the Acinetobacter calcoaceticus-Acinetobacter baumannii complex. J. Clin. Microbiol. 1992, 30, 2680-2685. [CrossRef]

12. Nemec, A.; Krizova, L.; Maixnerova, M.; Sedo, O.; Brisse, S.; Higgins, P.G. Acinetobacter seifertii sp. nov., a member of the Acinetobacter calcoaceticus-Acinetobacter baumannii complex isolated from human clinical specimens. Int. J. Syst. Evol. Microbiol. 2015, 65, 934-942. [CrossRef] [PubMed]

13. Cosgaya, C.; Marí-Almirall, M.; Van Assche, A.; Fernández-Orth, D.; Mosqueda, N.; Telli, M.; Huys, G.; Higgins, P.G.; Seifert, H.; Lievens, B.; et al. Acinetobacter dijkshoorniae sp. nov., a member of the Acinetobacter calcoaceticus-Acinetobacter baumannii complex mainly recovered from clinical samples in different countries. Int. J. Syst. Evol. Microbiol. 2016, 66, 4105-4111. [CrossRef] [PubMed] 
14. Li, Y.; Yang, X.; Zhao, W. Emerging Microtechnologies and Automated Systems for Rapid Bacterial Identification and Antibiotic Susceptibility Testing. SLAS Technol. 2017, 22, 585-608. [CrossRef] [PubMed]

15. Tjernberg, I.; Ursing, J. Clinical strains of Acinetobacter classified by DNA-DNA hybridization. APMIS Acta Pathol. Microbiol. Immunol. Scand. 1989, 97, 595-605. [CrossRef]

16. Misbah, S.; Hassan, H.; Yusof, M.Y.; Hanifah, Y.A.; AbuBakar, S. Genomic species identification of Acinetobacter of clinical isolates by $16 \mathrm{~S}$ rDNA sequencing. Singap. Med. J. 2005, 46, 461-464.

17. Šedo, O.; Nemec, A.; Kř́žová, L.; Kačalová, M.; Zdráhal, Z. Improvement of MALDI-TOF MS profiling for the differentiation of species within the Acinetobacter calcoaceticus-Acinetobacter baumannii complex. Syst. Appl. Microbiol. 2013, 36, 572-578. [CrossRef]

18. Rafei, R.; Osman, M.; Dabboussi, F.; Hamze, M. Update on the epidemiological typing methods for Acinetobacter baumannii. Future Microbiol. 2019, 14, 1065-1080. [CrossRef]

19. Fitzpatrick, M.A.; Ozer, E.A.; Hauser, A.R. Utility of Whole-Genome Sequencing in Characterizing Acinetobacter Epidemiology and Analyzing Hospital Outbreaks. J. Clin. Microbiol. 2016, 54, 593-612. [CrossRef]

20. Evans, B.A.; Amyes, S.G.B. OXA $\beta$-lactamases. Clin. Microbiol. Rev. 2014, 27, 241-263. [CrossRef]

21. Turton, J.F.; Woodford, N.; Glover, J.; Yarde, S.; Kaufmann, M.E.; Pitt, T.L. Identification of Acinetobacter baumannii by detection of the blaOXA-51-like carbapenemase gene intrinsic to this species. J. Clin. Microbiol. 2006, 44, 2974-2976. [CrossRef]

22. Antunes, L.C.S.; Visca, P.; Towner, K.J. Acinetobacter baumannii: Evolution of a global pathogen. Pathog. Dis. 2014, 71, 292-301. [CrossRef] [PubMed]

23. Djordjevic, Z.M.; Folic, M.M.; Folic, N.D.; Gajovic, N.; Gajovic, O.; Jankovic, S.M. Risk factors for hospital infections caused by carbapanem-resistant Acinetobacter baumannii. J. Infect. Dev. Ctries. 2016, 10, 1073-1080. [CrossRef] [PubMed]

24. Baran, G.; Erbay, A.; Bodur, H.; Ongürü, P.; Akinci, E.; Balaban, N.; Cevik, M.A. Risk factors for nosocomial imipenem-resistant Acinetobacter baumannii infections. Int. J. Infect. Dis. 2008, 12, 16-21. [CrossRef] [PubMed]

25. Lee, H.-Y.; Hsu, S.-Y.; Hsu, J.-F.; Chen, C.-L.; Wang, Y.-H.; Chiu, C.-H. Risk factors and molecular epidemiology of Acinetobacter baumannii bacteremia in neonates. J. Microbiol. Immunol. Infect. 2018, 51, 367-376. [CrossRef] [PubMed]

26. Fukuta, Y.; Muder, R.R.; Agha, M.E.; Clarke, L.G.; Wagener, M.M.; Hensler, A.M.; Doi, Y. Risk factors for acquisition of multidrug-resistant Acinetobacter baumannii among cancer patients. Am. J. Infect. Control 2013, 41, 1249-1252. [CrossRef] [PubMed]

27. Jaruratanasirikul, S.; Nitchot, W.; Wongpoowarak, W.; Samaeng, M.; Nawakitrangsan, M. Population pharmacokinetics and Monte Carlo simulations of sulbactam to optimize dosage regimens in patients with ventilator-associated pneumonia caused by Acinetobacter baumannii. Eur. J. Pharm. Sci. 2019, 136, 104940. [CrossRef] [PubMed]

28. Lynch, J.P.; Zhanel, G.G.; Clark, N.M. Infections Due to Acinetobacter baumannii in the ICU: Treatment Options. Semin. Respir. Crit. Care Med. 2017, 38, 311-325.

29. Huang, Y.; Zhou, Q.; Wang, W.; Huang, Q.; Liao, J.; Li, J.; Long, L.; Ju, T.; Zhang, Q.; Wang, H.; et al. Acinetobacter baumannii Ventilator-Associated Pneumonia: Clinical Efficacy of Combined Antimicrobial Therapy and in vitro Drug Sensitivity Test Results. Front. Pharmacol. 2019, 10, 92. [CrossRef]

30. Kanafani, Z.A.; Zahreddine, N.; Tayyar, R.; Sfeir, J.; Araj, G.F.; Matar, G.M.; Kanj, S.S. Multi-drug resistant Acinetobacter species: A seven-year experience from a tertiary care center in Lebanon. Antimicrob. Resist. Infect. Control 2018, 7, 9. [CrossRef]

31. Čiginskienè, A.; Dambrauskienè, A.; Rello, J.; Adukauskienè, D. Ventilator-Associated Pneumonia due to Drug-Resistant Acinetobacter baumannii: Risk Factors and Mortality Relation with Resistance Profiles, and Independent Predictors of In-Hospital Mortality. Medicina 2019, 55, 49. [CrossRef]

32. Nowak, J.; Zander, E.; Stefanik, D.; Higgins, P.G.; Roca, I.; Vila, J.; McConnell, M.J.; Cisneros, J.M.; Seifert, H. MagicBullet Working Group WP4 High incidence of pandrug-resistant Acinetobacter baumannii isolates collected from patients with ventilator-associated pneumonia in Greece, Italy and Spain as part of the MagicBullet clinical trial. J. Antimicrob. Chemother. 2017, 72, 3277-3282. [CrossRef] [PubMed] 
33. Mohd Sazlly Lim, S.; Zainal Abidin, A.; Liew, S.M.; Roberts, J.A.; Sime, F.B. The global prevalence of multidrug-resistance among Acinetobacter baumannii causing hospital-acquired and ventilator-associated pneumonia and its associated mortality: A systematic review and meta-analysis. J. Infect. 2019, 79, 593-600. [CrossRef] [PubMed]

34. Hoang Quoc, C.; Nguyen Thi Phuong, T.; Nguyen Duc, H.; Tran Le, T.; Tran Thi Thu, H.; Nguyen Tuan, S.; Phan Trong, L. Carbapenemase Genes and Multidrug Resistance of Acinetobacter baumannii: A Cross Sectional Study of Patients with Pneumonia in Southern Vietnam. Antibiotics 2019, 8, 148. [CrossRef] [PubMed]

35. Dexter, C.; Murray, G.L.; Paulsen, I.T.; Peleg, A.Y. Community-acquired Acinetobacter baumannii: Clinical characteristics, epidemiology and pathogenesis. Expert Rev. Anti-Infect. Ther. 2015, 13, 567-573. [CrossRef] [PubMed]

36. Wong, D.; Nielsen, T.B.; Bonomo, R.A.; Pantapalangkoor, P.; Luna, B.; Spellberg, B. Clinical and Pathophysiological Overview of Acinetobacter Infections: A Century of Challenges. Clin. Microbiol. Rev. 2017, 30, 409-447. [PubMed]

37. Jia, H.; Sun, Q.; Ruan, Z.; Xie, X. Characterization of a small plasmid carrying the carbapenem resistance gene blaOXA-72 from community-acquired Acinetobacter baumannii sequence type 880 in China. Infect. Drug Resist. 2019, 12, 1545-1553. [CrossRef]

38. Wisplinghoff, H.; Paulus, T.; Lugenheim, M.; Stefanik, D.; Higgins, P.G.; Edmond, M.B.; Wenzel, R.P.; Seifert, H. Nosocomial bloodstream infections due to Acinetobacter baumannii, Acinetobacter pittii and Acinetobacter nosocomialis in the United States. J. Infect. 2012, 64, 282-290. [CrossRef]

39. Freire, M.P.; de Oliveira Garcia, D.; Garcia, C.P.; Campagnari Bueno, M.F.; Camargo, C.H.; Kono Magri, A.S.G.; Francisco, G.R.; Reghini, R.; Vieira, M.F.; Ibrahim, K.Y.; et al. Bloodstream infection caused by extensively drug-resistant Acinetobacter baumannii in cancer patients: High mortality associated with delayed treatment rather than with the degree of neutropenia. Clin. Microbiol. Infect. 2016, 22, 352-358. [CrossRef]

40. Tsitsopoulos, P.P.; Iosifidis, E.; Antachopoulos, C.; Anestis, D.M.; Karantani, E.; Karyoti, A.; Papaevangelou, G.; Kyriazidis, E.; Roilides, E.; Tsonidis, C. Nosocomial bloodstream infections in neurosurgery: A 10-year analysis in a center with high antimicrobial drug-resistance prevalence. Acta Neurochir. (Wien) 2016, 158, 1647-1654. [CrossRef]

41. Gong, Y.L.; Yang, Z.C.; Yin, S.P.; Liu, M.X.; Zhang, C.; Luo, X.Q.; Peng, Y.Z. Analysis of the pathogenic characteristics of 162 severely burned patients with bloodstream infection. Chin. J. Burns 2016, 32, 529-535.

42. Motbainor, H.; Bereded, F.; Mulu, W. Multi-drug resistance of blood stream, urinary tract and surgical site nosocomial infections of Acinetobacter baumannii and Pseudomonas aeruginosa among patients hospitalized at Felegehiwot referral hospital, Northwest Ethiopia: A cross-sectional study. BMC Infect. Dis. 2020, $20,92$. [CrossRef] [PubMed]

43. Papathanakos, G.; Andrianopoulos, I.; Papathanasiou, A.; Priavali, E.; Koulenti, D.; Koulouras, V. Colistin-Resistant Acinetobacter baumannii Bacteremia: A Serious Threat for Critically Ill Patients. Microorganisms 2020, 8, 287. [CrossRef] [PubMed]

44. Davis, K.A.; Moran, K.A.; McAllister, C.K.; Gray, P.J. Multidrug-resistant Acinetobacter extremity infections in soldiers. Emerg. Infect. Dis. 2005, 11, 1218-1224. [CrossRef] [PubMed]

45. Johnson, E.N.; Burns, T.C.; Hayda, R.A.; Hospenthal, D.R.; Murray, C.K. Infectious complications of open type III tibial fractures among combat casualties. Clin. Infect. Dis. 2007, 45, 409-415. [CrossRef] [PubMed]

46. Keen, E.F.; Murray, C.K.; Robinson, B.J.; Hospenthal, D.R.; Co, E.-M.A.; Aldous, W.K. Changes in the incidences of multidrug-resistant and extensively drug-resistant organisms isolated in a military medical center. Infect. Control Hosp. Epidemiol. 2010, 31, 728-732. [CrossRef] [PubMed]

47. Albrecht, M.C.; Albrecht, M.A.; Griffith, M.E.; Murray, C.K.; Chung, K.K.; Horvath, E.E.; Ward, J.A.; Hospenthal, D.R.; Holcomb, J.B.; Wolf, S.E. Impact of Acinetobacter infection on the mortality of burn patients. J. Am. Coll. Surg. 2006, 203, 546-550. [CrossRef]

48. Dallo, S.F.; Weitao, T. Insights into acinetobacter war-wound infections, biofilms, and control. Adv. Skin Wound Care 2010, 23, 169-174. [CrossRef]

49. De Carvalho, V.C.; de Oliveira, P.R.D.; Dal-Paz, K.; de Paula, A.P.; Félix, C.D.S.; Lima, A.L.L.M. Gram-negative osteomyelitis: Clinical and microbiological profile. Braz. J. Infect. Dis. 2012, 16, 63-67. [CrossRef]

50. Zhang, B.; Liu, Z.; Lin, Z.; Zhang, X.; Fu, W. Microbiologic characteristics of pathogenic bacteria from hospitalized trauma patients who survived Wenchuan earthquake. Eur. J. Clin. Microbiol. Infect. Dis. 2012, 31, 2529-2535. [CrossRef] 
51. Di Venanzio, G.; Flores-Mireles, A.L.; Calix, J.J.; Haurat, M.F.; Scott, N.E.; Palmer, L.D.; Potter, R.F.; Hibbing, M.E.; Friedman, L.; Wang, B.; et al. Urinary tract colonization is enhanced by a plasmid that regulates uropathogenic Acinetobacter baumannii chromosomal genes. Nat. Commun. 2019, 10, 2763. [CrossRef]

52. Gaynes, R.; Edwards, J.R. National Nosocomial Infections Surveillance System Overview of nosocomial infections caused by gram-negative bacilli. Clin. Infect. Dis. 2005, 41, 848-854. [PubMed]

53. Balfousias, T.; Apostolopoulos, A.; Angelis, S.; Filippou, D.; Maris, S. Pandrug-resistant Acinetobacter baumannii Infection Identified in a Non-intensive Care Unit Patient: A Case Study. Cureus 2019, 11, e6321. [CrossRef] [PubMed]

54. Park, Y.K.; Lee, G.H.; Baek, J.Y.; Chung, D.R.; Peck, K.R.; Song, J.-H.; Ko, K.S. A single clone of Acinetobacter baumannii, ST22, is responsible for high antimicrobial resistance rates of Acinetobacter spp. isolates that cause bacteremia and urinary tract infections in Korea. Microb. Drug Resist. 2010, 16, 143-149. [CrossRef]

55. Siegman-Igra, Y.; Bar-Yosef, S.; Gorea, A.; Avram, J. Nosocomial acinetobacter meningitis secondary to invasive procedures: Report of 25 cases and review. Clin. Infect. Dis. 1993, 17, 843-849. [CrossRef] [PubMed]

56. Sharma, R.; Goda, R.; Borkar, S.A.; Katiyar, V.; Agarwal, S.; Kumar, A.; Mohapatra, S.; Kapil, A.; Suri, A.; Kale, S.S. Outcome following postneurosurgical Acinetobacter meningitis: An institutional experience of 72 cases. Neurosurg. Focus 2019, 47, E8. [CrossRef] [PubMed]

57. Xiao, J.; Zhang, C.; Ye, S. Acinetobacter baumannii meningitis in children: A case series and literature review. Infection 2019, 47, 643-649. [CrossRef]

58. Pan, S.; Huang, X.; Wang, Y.; Li, L.; Zhao, C.; Yao, Z.; Cui, W.; Zhang, G. Efficacy of intravenous plus intrathecal/intracerebral ventricle injection of polymyxin B for post-neurosurgical intracranial infections due to MDR/XDR Acinectobacter baumannii: A retrospective cohort study. Antimicrob. Resist. Infect. Control 2018, 7, 8. [CrossRef]

59. Smith, M.G.; Gianoulis, T.A.; Pukatzki, S.; Mekalanos, J.J.; Ornston, L.N.; Gerstein, M.; Snyder, M. New insights into Acinetobacter baumannii pathogenesis revealed by high-density pyrosequencing and transposon mutagenesis. Genes Dev. 2007, 21, 601-614. [CrossRef]

60. Sahl, J.W.; Johnson, J.K.; Harris, A.D.; Phillippy, A.M.; Hsiao, W.W.; Thom, K.A.; Rasko, D.A. Genomic comparison of multi-drug resistant invasive and colonizing Acinetobacter baumannii isolated from diverse human body sites reveals genomic plasticity. BMC Genom. 2011, 12, 291. [CrossRef]

61. Lin, J.; Huang, S.; Zhang, Q. Outer membrane proteins: Key players for bacterial adaptation in host niches. Microbes Infect. 2002, 4, 325-331. [CrossRef]

62. Choi, C.H.; Lee, E.Y.; Lee, Y.C.; Park, T.I.; Kim, H.J.; Hyun, S.H.; Kim, S.A.; Lee, S.-K.; Lee, J.C. Outer membrane protein 38 of Acinetobacter baumannii localizes to the mitochondria and induces apoptosis of epithelial cells. Cell. Microbiol. 2005, 7, 1127-1138. [CrossRef] [PubMed]

63. Choi, C.H.; Lee, J.S.; Lee, Y.C.; Park, T.I.; Lee, J.C. Acinetobacter baumannii invades epithelial cells and outer membrane protein A mediates interactions with epithelial cells. BMC Microbiol. 2008, 8, 216. [CrossRef] [PubMed]

64. Smani, Y.; McConnell, M.J.; Pachón, J. Role of fibronectin in the adhesion of Acinetobacter baumannii to host cells. PLoS ONE 2012, 7, e33073. [CrossRef] [PubMed]

65. Smani, Y.; Dominguez-Herrera, J.; Pachón, J. Association of the outer membrane protein Omp33 with fitness and virulence of Acinetobacter baumannii. J. Infect. Dis. 2013, 208, 1561-1570. [CrossRef]

66. Rumbo, C.; Tomás, M.; Fernández Moreira, E.; Soares, N.C.; Carvajal, M.; Santillana, E.; Beceiro, A.; Romero, A.; Bou, G. The Acinetobacter baumannii Omp33-36 porin is a virulence factor that induces apoptosis and modulates autophagy in human cells. Infect. Immun. 2014, 82, 4666-4680. [CrossRef]

67. Sato, Y.; Unno, Y.; Kawakami, S.; Ubagai, T.; Ono, Y. Virulence characteristics of Acinetobacter baumannii clinical isolates vary with the expression levels of omps. J. Med. Microbiol. 2017, 66, 203-212. [CrossRef]

68. Knapp, S.; Wieland, C.W.; Florquin, S.; Pantophlet, R.; Dijkshoorn, L.; Tshimbalanga, N.; Akira, S.; van der Poll, T. Differential roles of CD14 and toll-like receptors 4 and 2 in murine Acinetobacter pneumonia. Am. J. Respir. Crit. Care Med. 2006, 173, 122-129. [CrossRef]

69. Haseley, S.R.; Pantophlet, R.; Brade, L.; Holst, O.; Brade, H. Structural and serological characterisation of the O-antigenic polysaccharide of the lipopolysaccharide from Acinetobacter junii strain 65. Eur. J. Biochem. 1997, 245, 477-481. [CrossRef] 
70. Singh, J.K.; Adams, F.G.; Brown, M.H. Diversity and Function of Capsular Polysaccharide in Acinetobacter baumannii. Front. Microbiol. 2018, 9, 3301. [CrossRef]

71. Geisinger, E.; Isberg, R.R. Antibiotic modulation of capsular exopolysaccharide and virulence in Acinetobacter baumannii. PLoS Pathog. 2015, 11, e1004691. [CrossRef]

72. Kenyon, J.J.; Hall, R.M. Variation in the complex carbohydrate biosynthesis loci of Acinetobacter baumannii genomes. PLoS ONE 2013, 8, e62160. [CrossRef] [PubMed]

73. Camarena, L.; Bruno, V.; Euskirchen, G.; Poggio, S.; Snyder, M. Molecular mechanisms of ethanol-induced pathogenesis revealed by RNA-sequencing. PLoS Pathog. 2010, 6, e1000834. [CrossRef] [PubMed]

74. Waack, U.; Warnock, M.; Yee, A.; Huttinger, Z.; Smith, S.; Kumar, A.; Deroux, A.; Ginsburg, D.; Mobley, H.L.T.; Lawrence, D.A.; et al. CpaA Is a Glycan-Specific Adamalysin-like Protease Secreted by Acinetobacter baumannii That Inactivates Coagulation Factor XII. mBio 2018, 9, e01606-18. [CrossRef] [PubMed]

75. Giannouli, M.; Antunes, L.C.S.; Marchetti, V.; Triassi, M.; Visca, P.; Zarrilli, R. Virulence-related traits of epidemic Acinetobacter baumannii strains belonging to the international clonal lineages I-III and to the emerging genotypes ST25 and ST78. BMC Infect. Dis. 2013, 13, 282. [CrossRef]

76. Ophir, T.; Gutnick, D.L. A role for exopolysaccharides in the protection of microorganisms from desiccation. Appl. Environ. Microbiol. 1994, 60, 740-745. [CrossRef]

77. Boll, J.M.; Tucker, A.T.; Klein, D.R.; Beltran, A.M.; Brodbelt, J.S.; Davies, B.W.; Trent, M.S. Reinforcing Lipid A Acylation on the Cell Surface of Acinetobacter baumannii Promotes Cationic Antimicrobial Peptide Resistance and Desiccation Survival. mBio 2015, 6, e00478-415. [CrossRef]

78. Hassan, K.A.; Jackson, S.M.; Penesyan, A.; Patching, S.G.; Tetu, S.G.; Eijkelkamp, B.A.; Brown, M.H.; Henderson, P.J.F.; Paulsen, I.T. Transcriptomic and biochemical analyses identify a family of chlorhexidine efflux proteins. Proc. Natl. Acad. Sci. USA 2013, 110, 20254-20259. [CrossRef]

79. Nwugo, C.C.; Arivett, B.A.; Zimbler, D.L.; Gaddy, J.A.; Richards, A.M.; Actis, L.A. Effect of ethanol on differential protein production and expression of potential virulence functions in the opportunistic pathogen Acinetobacter baumannii. PLoS ONE 2012, 7, e51936. [CrossRef]

80. Asplund, M.B.; Coelho, C.; Cordero, R.J.B.; Martinez, L.R. Alcohol impairs J774.16 macrophage-like cell antimicrobial functions in Acinetobacter baumannii infection. Virulence 2013, 4, 467-472. [CrossRef]

81. Doi, Y.; Murray, G.L.; Peleg, A.Y. Acinetobacter baumannii: Evolution of antimicrobial resistance-treatment options. Semin. Respir. Crit. Care Med. 2015, 36, 85-98.

82. Greene, C.; Wu, J.; Rickard, A.H.; Xi, C. Evaluation of the ability of Acinetobacter baumannii to form biofilms on six different biomedical relevant surfaces. Lett. Appl. Microbiol. 2016, 63, 233-239. [CrossRef] [PubMed]

83. Tomaras, A.P.; Flagler, M.J.; Dorsey, C.W.; Gaddy, J.A.; Actis, L.A. Characterization of a two-component regulatory system from Acinetobacter baumannii that controls biofilm formation and cellular morphology. Microbiol. Read. Engl. 2008, 154, 3398-3409. [CrossRef] [PubMed]

84. Loehfelm, T.W.; Luke, N.R.; Campagnari, A.A. Identification and characterization of an Acinetobacter baumannii biofilm-associated protein. J. Bacteriol. 2008, 190, 1036-1044. [CrossRef] [PubMed]

85. Goh, H.M.S.; Beatson, S.A.; Totsika, M.; Moriel, D.G.; Phan, M.-D.; Szubert, J.; Runnegar, N.; Sidjabat, H.E.; Paterson, D.L.; Nimmo, G.R.; et al. Molecular analysis of the Acinetobacter baumannii biofilm-associated protein. Appl. Environ. Microbiol. 2013, 79, 6535-6543. [CrossRef]

86. Choi, A.H.K.; Slamti, L.; Avci, F.Y.; Pier, G.B.; Maira-Litrán, T. The pgaABCD locus of Acinetobacter baumannii encodes the production of poly-beta-1-6-N-acetylglucosamine, which is critical for biofilm formation. J. Bacteriol. 2009, 191, 5953-5963. [CrossRef]

87. Whiteley, M.; Diggle, S.P.; Greenberg, E.P. Progress in and promise of bacterial quorum sensing research. Nature 2017, 551, 313-320. [CrossRef]

88. Saipriya, K.; Swathi, C.H.; Ratnakar, K.S.; Sritharan, V. Quorum-sensing system in Acinetobacter baumannii: A potential target for new drug development. J. Appl. Microbiol. 2019, 128, 15-27. [CrossRef]

89. Alarcon, I.; Evans, D.J.; Fleiszig, S.M.J. The role of twitching motility in Pseudomonas aeruginosa exit from and translocation of corneal epithelial cells. Investig. Ophthalmol. Vis. Sci. 2009, 50, 2237-2244. [CrossRef]

90. Skiebe, E.; de Berardinis, V.; Morczinek, P.; Kerrinnes, T.; Faber, F.; Lepka, D.; Hammer, B.; Zimmermann, O.; Ziesing, S.; Wichelhaus, T.A.; et al. Surface-associated motility, a common trait of clinical isolates of Acinetobacter baumannii, depends on 1,3-diaminopropane. Int. J. Med. Microbiol. IJMM 2012, 302, 117-128. [CrossRef] 
91. Vijayakumar, S.; Rajenderan, S.; Laishram, S.; Anandan, S.; Balaji, V.; Biswas, I. Biofilm Formation and Motility Depend on the Nature of the Acinetobacter baumannii Clinical Isolates. Front. Public Health 2016, 4, 105. [CrossRef]

92. Harding, C.M.; Tracy, E.N.; Carruthers, M.D.; Rather, P.N.; Actis, L.A.; Munson, R.S. Acinetobacter baumannii strain M2 produces type IV pili which play a role in natural transformation and twitching motility but not surface-associated motility. mBio 2013, 4, e00360-13. [CrossRef] [PubMed]

93. Wilharm, G.; Piesker, J.; Laue, M.; Skiebe, E. DNA uptake by the nosocomial pathogen Acinetobacter baumannii occurs during movement along wet surfaces. J. Bacteriol. 2013, 195, 4146-4153. [CrossRef] [PubMed]

94. Burrows, L.L. Pseudomonas aeruginosa twitching motility: Type IV pili in action. Annu. Rev. Microbiol. 2012, 66, 493-520. [CrossRef] [PubMed]

95. Eijkelkamp, B.A.; Stroeher, U.H.; Hassan, K.A.; Elbourne, L.D.H.; Paulsen, I.T.; Brown, M.H. H-NS plays a role in expression of Acinetobacter baumannii virulence features. Infect. Immun. 2013, 81, 2574-2583. [CrossRef]

96. Pérez-Varela, M.; Corral, J.; Vallejo, J.A.; Rumbo-Feal, S.; Bou, G.; Aranda, J.; Barbé, J. Mutations in the $\beta$-Subunit of the RNA Polymerase Impair the Surface-Associated Motility and Virulence of Acinetobacter baumannii. Infect. Immun. 2017, 85, e00327-17.

97. Ahmad, I.; Nygren, E.; Khalid, F.; Myint, S.L.; Uhlin, B.E. A Cyclic-di-GMP signalling network regulates biofilm formation and surface associated motility of Acinetobacter baumannii 17978. Sci. Rep. 2020, 10, 1991. [CrossRef]

98. Tipton, K.A.; Rather, P.N. An ompR-envZ Two-Component System Ortholog Regulates Phase Variation, Osmotic Tolerance, Motility, and Virulence in Acinetobacter baumannii Strain AB5075. J. Bacteriol. 2017, 199, e00705-16. [CrossRef]

99. Morris, F.C.; Dexter, C.; Kostoulias, X.; Uddin, M.I.; Peleg, A.Y. The Mechanisms of Disease Caused by Acinetobacter baumannii. Front. Microbiol. 2019, 10, 1601. [CrossRef]

100. Ajiboye, T.O.; Skiebe, E.; Wilharm, G. Contributions of ferric uptake regulator Fur to the sensitivity and oxidative response of Acinetobacter baumannii to antibiotics. Microb. Pathog. 2018, 119, 35-41. [CrossRef]

101. Moore, J.L.; Becker, K.W.; Nicklay, J.J.; Boyd, K.L.; Skaar, E.P.; Caprioli, R.M. Imaging mass spectrometry for assessing temporal proteomics: Analysis of calprotectin in Acinetobacter baumannii pulmonary infection. Proteomics 2014, 14, 820-828. [CrossRef]

102. Nairn, B.L.; Lonergan, Z.R.; Wang, J.; Braymer, J.J.; Zhang, Y.; Calcutt, M.W.; Lisher, J.P.; Gilston, B.A.; Chazin, W.J.; de Crécy-Lagard, V.; et al. The Response of Acinetobacter baumannii to Zinc Starvation. Cell Host Microbe 2016, 19, 826-836. [CrossRef] [PubMed]

103. Juttukonda, L.J.; Chazin, W.J.; Skaar, E.P. Acinetobacter baumannii Coordinates Urea Metabolism with Metal Import To Resist Host-Mediated Metal Limitation. mBio 2016, 7, e01475-16. [CrossRef] [PubMed]

104. Weber, B.S.; Kinsella, R.L.; Harding, C.M.; Feldman, M.F. The Secrets of Acinetobacter Secretion. Trends Microbiol. 2017, 25, 532-545. [CrossRef]

105. Bentancor, L.V.; Camacho-Peiro, A.; Bozkurt-Guzel, C.; Pier, G.B.; Maira-Litrán, T. Identification of Ata, a multifunctional trimeric autotransporter of Acinetobacter baumannii. J. Bacteriol. 2012, 194, 3950-3960. [CrossRef] [PubMed]

106. Sandkvist, M. Type II secretion and pathogenesis. Infect. Immun. 2001, 69, 3523-3535. [CrossRef] [PubMed]

107. Tilley, D.; Law, R.; Warren, S.; Samis, J.A.; Kumar, A. CpaA a novel protease from Acinetobacter baumannii clinical isolates deregulates blood coagulation. FEMS Microbiol. Lett. 2014, 356, 53-61. [CrossRef]

108. Hood, R.D.; Singh, P.; Hsu, F.; Güvener, T.; Carl, M.A.; Trinidad, R.R.S.; Silverman, J.M.; Ohlson, B.B.; Hicks, K.G.; Plemel, R.L.; et al. A type VI secretion system of Pseudomonas aeruginosa targets a toxin to bacteria. Cell Host Microbe 2010, 7, 25-37. [CrossRef]

109. Elhosseiny, N.M.; Attia, A.S. Acinetobacter: An emerging pathogen with a versatile secretome. Emerg. Microbes Infect. 2018, 7, 33. [CrossRef]

110. Repizo, G.D. Prevalence of Acinetobacter baumannii strains expressing the Type 6 secretion system in patients with bacteremia. Virulence 2017, 8, 1099-1101. [CrossRef]

111. Harding, C.M.; Hennon, S.W.; Feldman, M.F. Uncovering the mechanisms of Acinetobacter baumannii virulence. Nat. Rev. Microbiol. 2018, 16, 91-102. [CrossRef]

112. Fishbain, J.; Peleg, A.Y. Treatment of Acinetobacter infections. Clin. Infect. Dis. 2010, 51, 79-84. [CrossRef] [PubMed] 
113. Garnacho-Montero, J.; Ortiz-Leyba, C.; Jiménez-Jiménez, F.J.; Barrero-Almodóvar, A.E.; García-Garmendia, J.L.; Bernabeu-WittelI, M.; Gallego-Lara, S.L.; Madrazo-Osuna, J. Treatment of multidrug-resistant Acinetobacter baumannii ventilator-associated pneumonia (VAP) with intravenous colistin: A comparison with imipenem-susceptible VAP. Clin. Infect. Dis. 2003, 36, 1111-1118. [CrossRef] [PubMed]

114. Superti, S.V.; Martins, D.D.S.; Caierão, J.; Soares, F.D.S.; Prochnow, T.; Zavascki, A.P. Indications of carbapenem resistance evolution through heteroresistance as an intermediate stage in Acinetobacter baumannii after carbapenem administration. Rev. Inst. Med. Trop. Sao Paulo 2009, 51, 111-113. [CrossRef] [PubMed]

115. Falagas, M.E.; Kasiakou, S.K. Toxicity of polymyxins: A systematic review of the evidence from old and recent studies. Crit. Care Lond. Engl. 2006, 10, R27. [CrossRef] [PubMed]

116. Ritchie, D.J.; Garavaglia-Wilson, A. A review of intravenous minocycline for treatment of multidrug-resistant Acinetobacter infections. Clin. Infect. Dis. 2014, 59 (Suppl. 6), S374-S380. [CrossRef]

117. Lee, Y.-T.; Tsao, S.-M.; Hsueh, P.-R. Clinical outcomes of tigecycline alone or in combination with other antimicrobial agents for the treatment of patients with healthcare-associated multidrug-resistant Acinetobacter baumannii infections. Eur. J. Clin. Microbiol. Infect. Dis. 2013, 32, 1211-1220. [CrossRef]

118. Karageorgopoulos, D.E.; Kelesidis, T.; Kelesidis, I.; Falagas, M.E. Tigecycline for the treatment of multidrug-resistant (including carbapenem-resistant) Acinetobacter infections: A review of the scientific evidence. J. Antimicrob. Chemother. 2008, 62, 45-55. [CrossRef]

119. Freire, A.T.; Melnyk, V.; Kim, M.J.; Datsenko, O.; Dzyublik, O.; Glumcher, F.; Chuang, Y.-C.; Maroko, R.T.; Dukart, G.; Cooper, C.A.; et al. Comparison of tigecycline with imipenem/cilastatin for the treatment of hospital-acquired pneumonia. Diagn. Microbiol. Infect. Dis. 2010, 68, 140-151. [CrossRef]

120. Penwell, W.F.; Shapiro, A.B.; Giacobbe, R.A.; Gu, R.-F.; Gao, N.; Thresher, J.; McLaughlin, R.E.; Huband, M.D.; DeJonge, B.L.M.; Ehmann, D.E.; et al. Molecular mechanisms of sulbactam antibacterial activity and resistance determinants in Acinetobacter baumannii. Antimicrob. Agents Chemother. 2015, 59, 1680-1689. [CrossRef]

121. Rigatto, M.H.; Vieira, F.J.; Antochevis, L.C.; Behle, T.F.; Lopes, N.T.; Zavascki, A.P. Polymyxin B in Combination with Antimicrobials Lacking In Vitro Activity versus Polymyxin B in Monotherapy in Critically Ill Patients with Acinetobacter baumannii or Pseudomonas aeruginosa Infections. Antimicrob. Agents Chemother. 2015, 59, 6575-6580. [CrossRef]

122. Durante-Mangoni, E.; Signoriello, G.; Andini, R.; Mattei, A.; De Cristoforo, M.; Murino, P.; Bassetti, M.; Malacarne, P.; Petrosillo, N.; Galdieri, N.; et al. Colistin and rifampicin compared with colistin alone for the treatment of serious infections due to extensively drug-resistant Acinetobacter baumannii: A multicenter, randomized clinical trial. Clin. Infect. Dis. 2013, 57, 349-358. [CrossRef] [PubMed]

123. Spellberg, B.; Bonomo, R.A. Combination Therapy for Extreme Drug-Resistant Acinetobacter baumannii: Ready for Prime Time? Crit. Care Med. 2015, 43, 1332-1334. [CrossRef] [PubMed]

124. Le Minh, V.; Thi Khanh Nhu, N.; Vinh Phat, V.; Thompson, C.; Huong Lan, N.P.; Thieu Nga, T.V.; Thanh Tam, P.T.; Tuyen, H.T.; Hoang Nhu, T.D.; Van Hao, N.; et al. In vitro activity of colistin in antimicrobial combination against carbapenem-resistant Acinetobacter baumannii isolated from patients with ventilator-associated pneumonia in Vietnam. J. Med. Microbiol. 2015, 64, 1162-1169. [CrossRef] [PubMed]

125. Oleksiuk, L.M.; Nguyen, M.H.; Press, E.G.; Updike, C.L.; O’Hara, J.A.; Doi, Y.; Clancy, C.J.; Shields, R.K. In vitro responses of Acinetobacter baumannii to two- and three-drug combinations following exposure to colistin and doripenem. Antimicrob. Agents Chemother. 2014, 58, 1195-1199. [CrossRef] [PubMed]

126. Shi, H.; Lee, J.S.; Park, S.Y.; Ko, Y.; Eom, J.S. Colistin Plus Carbapenem versus Colistin Monotherapy in the Treatment of Carbapenem-Resistant Acinetobacter baumannii Pneumonia. Infect. Drug Resist. 2019, 12, 3925-3934. [CrossRef]

127. Sertcelik, A.; Baran, I.; Akinci, E.; Mumcuoglu, I.; Bodur, H. Synergistic Activities of Colistin Combinations with Meropenem, Sulbactam, Minocycline, Disodium Fosfomycin, or Vancomycin Against Different Clones of Carbapenem-Resistant Acinetobacter baumannii Strains. Microb. Drug Resist. 2019. [CrossRef]

128. Hammoudi, D.; Moubareck, C.A.; Sarkis, D.K. How to detect carbapenemase producers? A literature review of phenotypic and molecular methods. J. Microbiol. Methods 2014, 107, 106-118. [CrossRef]

129. Alkasaby, N.M.; El Sayed Zaki, M. Molecular Study of Acinetobacter baumannii Isolates for Metallo- $\beta$-Lactamases and Extended-Spectrum- $\beta$-Lactamases Genes in Intensive Care Unit, Mansoura University Hospital, Egypt. Int. J. Microbiol. 2017, 2017, 3925868. [CrossRef] 
130. Pfeifer, Y.; Hunfeld, K.-P.; Borgmann, S.; Maneg, D.; Blobner, W.; Werner, G.; Higgins, P.G. Carbapenem-resistant Acinetobacter baumannii ST78 with OXA-72 carbapenemase and ESBL gene blaCTX-M-115. J. Antimicrob. Chemother. 2016, 71, 1426-1428. [CrossRef]

131. Uddin, F.; McHugh, T.D.; Roulston, K.; Platt, G.; Khan, T.A.; Sohail, M. Detection of carbapenemases, AmpC and ESBL genes in Acinetobacter isolates from ICUs by DNA microarray. J. Microbiol. Methods 2018, 155, 19-23. [CrossRef]

132. Safari, M.; Mozaffari Nejad, A.S.; Bahador, A.; Jafari, R.; Alikhani, M.Y. Prevalence of ESBL and MBL encoding genes in Acinetobacter baumannii strains isolated from patients of intensive care units (ICU). Saudi J. Biol. Sci. 2015, 22, 424-429. [CrossRef] [PubMed]

133. Lee, C.-R.; Lee, J.H.; Park, M.; Park, K.S.; Bae, I.K.; Kim, Y.B.; Cha, C.-J.; Jeong, B.C.; Lee, S.H. Biology of Acinetobacter baumannii: Pathogenesis, Antibiotic Resistance Mechanisms, and Prospective Treatment Options. Front. Cell. Infect. Microbiol. 2017, 7, 55. [CrossRef] [PubMed]

134. Poirel, L.; Corvec, S.; Rapoport, M.; Mugnier, P.; Petroni, A.; Pasteran, F.; Faccone, D.; Galas, M.; Drugeon, H.; Cattoir, V.; et al. Identification of the novel narrow-spectrum beta-lactamase SCO-1 in Acinetobacter spp. from Argentina. Antimicrob. Agents Chemother. 2007, 51, 2179-2184. [CrossRef] [PubMed]

135. Moubareck, C.; Brémont, S.; Conroy, M.-C.; Courvalin, P.; Lambert, T. GES-11, a novel integron-associated GES variant in Acinetobacter baumannii. Antimicrob. Agents Chemother. 2009, 53, 3579-3581. [CrossRef] [PubMed]

136. Hammoudi, D.; Moubareck, C.A.; Hakime, N.; Houmani, M.; Barakat, A.; Najjar, Z.; Suleiman, M.; Fayad, N.; Sarraf, R.; Sarkis, D.K. Spread of imipenem-resistant Acinetobacter baumannii co-expressing OXA-23 and GES-11 carbapenemases in Lebanon. Int. J. Infect. Dis. 2015, 36, 56-61. [CrossRef]

137. Robledo, I.E.; Aquino, E.E.; Santé, M.I.; Santana, J.L.; Otero, D.M.; León, C.F.; Vázquez, G.J. Detection of KPC in Acinetobacter spp. in Puerto Rico. Antimicrob. Agents Chemother. 2010, 54, 1354-1357. [CrossRef]

138. Caneiras, C.; Calisto, F.; Jorge da Silva, G.; Lito, L.; Melo-Cristino, J.; Duarte, A. First Description of Colistin and Tigecycline-Resistant Acinetobacter baumannii Producing KPC-3 Carbapenemase in Portugal. Antibiotics 2018, 7, 96. [CrossRef]

139. Ribeiro, P.C.S.; Monteiro, A.S.; Marques, S.G.; Monteiro, S.G.; Monteiro-Neto, V.; Coqueiro, M.M.M.; Marques, A.C.G.; de Jesus Gomes Turri, R.; Santos, S.G.; Bomfim, M.R.Q. Phenotypic and molecular detection of the bla KPC gene in clinical isolates from inpatients at hospitals in São Luis, MA, Brazil. BMC Infect. Dis. 2016, 16, 737. [CrossRef]

140. Lima, W.G.; Silva Alves, G.C.; Sanches, C.; Antunes Fernandes, S.O.; de Paiva, M.C. Carbapenem-resistant Acinetobacter baumannii in patients with burn injury: A systematic review and meta-analysis. Burns 2019, 45, 1495-1508. [CrossRef]

141. Martinez, T.; Martinez, I.; Vazquez, G.J.; Aquino, E.E.; Robledo, I.E. Genetic environment of the KPC gene in Acinetobacter baumannii ST2 clone from Puerto Rico and genomic insights into its drug resistance. J. Med. Microbiol. 2016, 65, 784-792. [CrossRef]

142. Queenan, A.M.; Bush, K. Carbapenemases: The versatile beta-lactamases. Clin. Microbiol. Rev. 2007, 20, 440-458. [CrossRef] [PubMed]

143. Chen, Y.; Zhou, Z.; Jiang, Y.; Yu, Y. Emergence of NDM-1-producing Acinetobacter baumannii in China. J. Antimicrob. Chemother. 2011, 66, 1255-1259. [CrossRef] [PubMed]

144. Jaidane, N.; Naas, T.; Oueslati, S.; Bernabeu, S.; Boujaafar, N.; Bouallegue, O.; Bonnin, R.A. Whole-genome sequencing of NDM-1-producing ST85 Acinetobacter baumannii isolates from Tunisia. Int. J. Antimicrob. Agents 2018, 52, 916-921. [CrossRef] [PubMed]

145. Beigverdi, R.; Sattari-Maraji, A.; Emaneini, M.; Jabalameli, F. Status of carbapenem-resistant Acinetobacter baumannii harboring carbapenemase: First systematic review and meta-analysis from Iran. Infect. Genet. Evol. 2019, 73, 433-443. [CrossRef]

146. Salloum, T.; Tannous, E.; Alousi, S.; Arabaghian, H.; Rafei, R.; Hamze, M.; Tokajian, S. Genomic mapping of ST85 blaNDM-1 and blaOXA-94 producing Acinetobacter baumannii isolates from Syrian Civil War Victims. Int. J. Infect. Dis. 2018, 74, 100-108. [CrossRef] [PubMed]

147. El-Mahdy, T.S.; Al-Agamy, M.H.; Al-Qahtani, A.A.; Shibl, A.M. Detection of blaOXA-23-like and blaNDM-1 in Acinetobacter baumannii from the Eastern Region, Saudi Arabia. Microb. Drug Resist. 2017, 23, 115-121. [CrossRef] 
148. Ramadan, R.A.; Gebriel, M.G.; Kadry, H.M.; Mosallem, A. Carbapenem-resistant Acinetobacter baumannii and Pseudomonas aeruginosa: Characterization of carbapenemase genes and E-test evaluation of colistin-based combinations. Infect. Drug Resist. 2018, 11, 1261-1269. [CrossRef]

149. Girija, S.A.; Jayaseelan, V.P.; Arumugam, P. Prevalence of VIM- and GIM-producing Acinetobacter baumannii from patients with severe urinary tract infection. Acta Microbiol. Immunol. Hung. 2018, 65, 539-550. [CrossRef]

150. Gholami, M.; Moshiri, M.; Ahanjan, M.; Salimi Chirani, A.; Hasannejad Bibalan, M.; Asadi, A.; Eshaghi, M.; Pournajaf, A.; Abbasian, S.; Kouhsari, E.; et al. The diversity of class B and class D carbapenemases in clinical Acinetobacter baumannii isolates. Infez. Med. 2018, 26, 329-335.

151. Aghamiri, S.; Amirmozafari, N.; Fallah Mehrabadi, J.; Fouladtan, B.; Hanafi Abdar, M. Antibiotic Resistance Patterns and a Survey of Metallo- $\beta$-Lactamase Genes Including bla-IMP and bla-VIM Types in Acinetobacter baumannii Isolated from Hospital Patients in Tehran. Chemotherapy 2016, 61, 275-280. [CrossRef]

152. Rezaei, A.; Fazeli, H.; Halaji, M.; Moghadampour, M.; Faghri, J. Prevalence of metallo-beta-lactamase producing Acinetobacter baumannii isolated from intensive care unit in tertiary care hospitals. Ann. Ig. Med. Prev. Comunita 2018, 30, 330-336.

153. Shakibaie, M.R.; Azizi, O.; Shahcheraghi, F. Insight into stereochemistry of a new IMP allelic variant (IMP-55) metallo- $\beta$-lactamase identified in a clinical strain of Acinetobacter baumannii. Infect. Genet. Evol. 2017, 51, 118-126. [CrossRef] [PubMed]

154. Leungtongkam, U.; Thummeepak, R.; Tasanapak, K.; Sitthisak, S. Acquisition and transfer of antibiotic resistance genes in association with conjugative plasmid or class 1 integrons of Acinetobacter baumannii. PLoS ONE 2018, 13, e0208468. [CrossRef] [PubMed]

155. Jeon, J.H.; Lee, J.H.; Lee, J.J.; Park, K.S.; Karim, A.M.; Lee, C.-R.; Jeong, B.C.; Lee, S.H. Structural basis for carbapenem-hydrolyzing mechanisms of carbapenemases conferring antibiotic resistance. Int. J. Mol. Sci. 2015, 16, 9654-9692. [CrossRef]

156. Héritier, C.; Poirel, L.; Nordmann, P. Cephalosporinase over-expression resulting from insertion of ISAba1 in Acinetobacter baumannii. Clin. Microbiol. Infect. 2006, 12, 123-130. [CrossRef]

157. Kumburu, H.H.; Sonda, T.; van Zwetselaar, M.; Leekitcharoenphon, P.; Lukjancenko, O.; Mmbaga, B.T.; Alifrangis, M.; Lund, O.; Aarestrup, F.M.; Kibiki, G.S. Using WGS to identify antibiotic resistance genes and predict antimicrobial resistance phenotypes in MDR Acinetobacter baumannii in Tanzania. J. Antimicrob. Chemother. 2019, 74, 1484-1493. [CrossRef]

158. Jia, H.; Chen, Y.; Wang, J.; Ruan, Z. Genomic characterisation of a clinical Acinetobacter baumannii ST1928 isolate carrying a new ampC allelic variant blaADC-196 gene from China. J. Glob. Antimicrob. Resist. 2019, 19, 43-45. [CrossRef]

159. Mayanskiy, N.; Chebotar, I.; Alyabieva, N.; Kryzhanovskaya, O.; Savinova, T.; Turenok, A.; Bocharova, Y.; Lazareva, A.; Polikarpova, S.; Karaseva, O. Emergence of the Uncommon Clone ST944/ST78 Carrying blaOXA-40-like and blaCTX-M-like Genes Among Carbapenem-Nonsusceptible Acinetobacter baumannii in Moscow, Russia. Microb. Drug Resist. 2017, 23, 864-870. [CrossRef]

160. Jiang, N.; Zhang, X.; Zhou, Y.; Zhang, Z.; Zheng, X. Whole-genome sequencing of an NDM-1- and OXA-58-producing Acinetobacter towneri isolate from hospital sewage in Sichuan Province, China. J. Glob. Antimicrob. Resist. 2019, 16, 4-5. [CrossRef]

161. Sarikhani, Z.; Nazari, R.; Nateghi Rostami, M. First report of OXA-143-lactamase producing Acinetobacter baumannii in Qom, Iran. Iran. J. Basic Med. Sci. 2017, 20, 1282-1286.

162. Higgins, P.G.; Pérez-Llarena, F.J.; Zander, E.; Fernández, A.; Bou, G.; Seifert, H. OXA-235, a novel class D $\beta$-lactamase involved in resistance to carbapenems in Acinetobacter baumannii. Antimicrob. Agents Chemother. 2013, 57, 2121-2126. [CrossRef] [PubMed]

163. Paton, R.; Miles, R.S.; Hood, J.; Amyes, S.G.; Miles, R.S.; Amyes, S.G. ARI 1: Beta-lactamase-mediated imipenem resistance in Acinetobacter baumannii. Int. J. Antimicrob. Agents 1993, 2, 81-87. [CrossRef]

164. Scaife, W.; Young, H.K.; Paton, R.H.; Amyes, S.G. Transferable imipenem-resistance in Acinetobacter species from a clinical source. J. Antimicrob. Chemother. 1995, 36, 585-586. [CrossRef] [PubMed]

165. Mugnier, P.D.; Poirel, L.; Naas, T.; Nordmann, P. Worldwide dissemination of the blaOXA-23 carbapenemase gene of Acinetobacter baumannii. Emerg. Infect. Dis. 2010, 16, 35-40. [CrossRef] 
166. Figueiredo, S.; Poirel, L.; Papa, A.; Koulourida, V.; Nordmann, P. Overexpression of the naturally occurring blaOXA-51 gene in Acinetobacter baumannii mediated by novel insertion sequence ISAba9. Antimicrob. Agents Chemother. 2009, 53, 4045-4047. [CrossRef]

167. Wibberg, D.; Salto, I.P.; Eikmeyer, F.G.; Maus, I.; Winkler, A.; Nordmann, P.; Pühler, A.; Poirel, L.; Schlüter, A. Complete Genome Sequencing of Acinetobacter baumannii Strain K50 Discloses the Large Conjugative Plasmid pK50a Encoding Carbapenemase OXA-23 and Extended-Spectrum $\beta$-Lactamase GES-11. Antimicrob. Agents Chemother. 2018, 62, e00212-18. [CrossRef]

168. Kumar, S.; Patil, P.P.; Singhal, L.; Ray, P.; Patil, P.B.; Gautam, V. Molecular epidemiology of carbapenem-resistant Acinetobacter baumannii isolates reveals the emergence of blaOXA-23 and blaNDM-1 encoding international clones in India. Infect. Genet. Evol. 2019, 75, 103986. [CrossRef]

169. Mathlouthi, N.; Ben Lamine, Y.; Somai, R.; Bouhalila-Besbes, S.; Bakour, S.; Rolain, J.-M.; Chouchani, C. Incidence of OXA-23 and OXA-58 Carbapenemases Coexpressed in Clinical Isolates of Acinetobacter baumannii in Tunisia. Microb. Drug Resist. 2018, 24, 136-141. [CrossRef]

170. Santimaleeworagun, W.; Samret, W.; Preechachuawong, P.; Kerdsin, A.; Jitwasinkul, T. Emergence of Co-Carbapenemase Genes, Bla(Oxa23), Bla(Vim) And Bla(Ndm) In Carbapenemresistant Acinetobacter baumannii Clinical Isolates. Southeast Asian J. Trop. Med. Public Health 2016, 47, 1001-1007.

171. Zhu, L.-J.; Chen, X.-Y.; Hou, P.-F. Mutation of CarO participates in drug resistance in imipenem-resistant Acinetobacter baumannii. J. Clin. Lab. Anal. 2019, 33, e22976. [CrossRef]

172. Benmahmod, A.B.; Said, H.S.; Ibrahim, R.H. Prevalence and Mechanisms of Carbapenem Resistance Among Acinetobacter baumannii Clinical Isolates in Egypt. Microb. Drug Resist. 2019, 25, 480-488. [CrossRef] [PubMed]

173. Cayô, R.; Rodríguez, M.-C.; Espinal, P.; Fernández-Cuenca, F.; Ocampo-Sosa, A.A.; Pascual, A.; Ayala, J.A.; Vila, J.; Martínez-Martínez, L. Analysis of genes encoding penicillin-binding proteins in clinical isolates of Acinetobacter baumannii. Antimicrob. Agents Chemother. 2011, 55, 5907-5913. [CrossRef] [PubMed]

174. Mirshekar, M.; Shahcheraghi, F.; Azizi, O.; Solgi, H.; Badmasti, F. Diversity of Class 1 Integrons, and Disruption of carO and dacD by Insertion Sequences among Acinetobacter baumannii Isolates in Tehran, Iran. Microb. Drug Resist. 2018, 24, 359-366. [CrossRef] [PubMed]

175. Rumbo, C.; Gato, E.; López, M.; Ruiz de Alegría, C.; Fernández-Cuenca, F.; Martínez-Martínez, L.; Vila, J.; Pachón, J.; Cisneros, J.M.; Rodríguez-Baño, J.; et al. Contribution of efflux pumps, porins, and $\beta$-lactamases to multidrug resistance in clinical isolates of Acinetobacter baumannii. Antimicrob. Agents Chemother. 2013, 57, 5247-5257. [CrossRef] [PubMed]

176. Xu, C.; Bilya, S.R.; Xu, W. adeABC efflux gene in Acinetobacter baumannii. New Microbes New Infect. 2019, 30, 100549. [CrossRef]

177. Marchand, I.; Damier-Piolle, L.; Courvalin, P.; Lambert, T. Expression of the RND-type efflux pump AdeABC in Acinetobacter baumannii is regulated by the AdeRS two-component system. Antimicrob. Agents Chemother. 2004, 48, 3298-3304. [CrossRef]

178. Dou, Q.; Zou, M.; Li, J.; Wang, H.; Hu, Y.; Liu, W. AdeABC efflux pump and resistance of Acinetobacter baumannii against carbapenem. Med. Sci. 2017, 42, 426-433.

179. Chopra, I.; Roberts, M. Tetracycline antibiotics: Mode of action, applications, molecular biology, and epidemiology of bacterial resistance. Microbiol. Mol. Biol. Rev. MMBR 2001, 65, 232-260. [CrossRef]

180. Huys, G.; Cnockaert, M.; Vaneechoutte, M.; Woodford, N.; Nemec, A.; Dijkshoorn, L.; Swings, J. Distribution of tetracycline resistance genes in genotypically related and unrelated multiresistant Acinetobacter baumannii strains from different European hospitals. Res. Microbiol. 2005, 156, 348-355. [CrossRef]

181. Magnet, S.; Courvalin, P.; Lambert, T. Resistance-nodulation-cell division-type efflux pump involved in aminoglycoside resistance in Acinetobacter baumannii strain BM4454. Antimicrob. Agents Chemother. 2001, 45, 3375-3380. [CrossRef]

182. Damier-Piolle, L.; Magnet, S.; Brémont, S.; Lambert, T.; Courvalin, P. AdeIJK, a resistance-nodulation-cell division pump effluxing multiple antibiotics in Acinetobacter baumannii. Antimicrob. Agents Chemother. 2008, 52, 557-562. [CrossRef] [PubMed]

183. Yuhan, Y.; Ziyun, Y.; Yongbo, Z.; Fuqiang, L.; Qinghua, Z. Over expression of AdeABC and AcrAB-TolC efflux systems confers tigecycline resistance in clinical isolates of Acinetobacter baumannii and Klebsiella pneumoniae. Rev. Soc. Bras. Med. Trop. 2016, 49, 165-171. [CrossRef] [PubMed] 
184. Savari, M.; Ekrami, A.; Shoja, S.; Bahador, A. Plasmid borne Carbapenem-Hydrolyzing Class D $\beta$-Lactamases (CHDLs) and AdeABC efflux pump conferring carbapenem-tigecycline resistance among Acinetobacter baumannii isolates harboring TnAbaRs. Microb. Pathog. 2017, 104, 310-317. [CrossRef] [PubMed]

185. Costello, S.E.; Gales, A.C.; Morfin-Otero, R.; Jones, R.N.; Castanheira, M. Mechanisms of Resistance, Clonal Expansion, and Increasing Prevalence of Acinetobacter baumannii Strains Displaying Elevated Tigecycline MIC Values in Latin America. Microb. Drug Resist. 2016, 22, 253-258. [CrossRef] [PubMed]

186. Livermore, D.M. Tigecycline: What is it, and where should it be used? J. Antimicrob. Chemother. 2005, 56, 611-614. [CrossRef] [PubMed]

187. Ribera, A.; Ruiz, J.; Vila, J. Presence of the Tet M determinant in a clinical isolate of Acinetobacter baumannii. Antimicrob. Agents Chemother. 2003, 47, 2310-2312. [CrossRef] [PubMed]

188. Dönhöfer, A.; Franckenberg, S.; Wickles, S.; Berninghausen, O.; Beckmann, R.; Wilson, D.N. Structural basis for TetM-mediated tetracycline resistance. Proc. Natl. Acad. Sci. USA 2012, 109, 16900-16905. [CrossRef]

189. Brown, P.C.; Borowska, E.; Schwartz, T.; Horn, H. Impact of the particulate matter from wastewater discharge on the abundance of antibiotic resistance genes and facultative pathogenic bacteria in downstream river sediments. Sci. Total Environ. 2019, 649, 1171-1178. [CrossRef]

190. Zhou, Q.; Wang, M.; Zhong, X.; Liu, P.; Xie, X.; Wangxiao, J.; Sun, Y. Dissemination of resistance genes in duck/fish polyculture ponds in Guangdong Province: Correlations between $\mathrm{Cu}$ and $\mathrm{Zn}$ and antibiotic resistance genes. Environ. Sci. Pollut. Res. Int. 2019, 26, 8182-8193. [CrossRef]

191. Perez, F.; Hujer, A.M.; Hujer, K.M.; Decker, B.K.; Rather, P.N.; Bonomo, R.A. Global challenge of multidrug-resistant Acinetobacter baumannii. Antimicrob. Agents Chemother. 2007, 51, 3471-3484. [CrossRef]

192. Hamouda, A.; Amyes, S.G.B. Novel gyrA and parC point mutations in two strains of Acinetobacter baumannii resistant to ciprofloxacin. J. Antimicrob. Chemother. 2004, 54, 695-696. [CrossRef] [PubMed]

193. Spence, R.P.; Towner, K.J. Frequencies and mechanisms of resistance to moxifloxacin in nosocomial isolates of Acinetobacter baumannii. J. Antimicrob. Chemother. 2003, 52, 687-690. [CrossRef] [PubMed]

194. Su, X.-Z.; Chen, J.; Mizushima, T.; Kuroda, T.; Tsuchiya, T. AbeM, an H+-coupled Acinetobacter baumannii multidrug efflux pump belonging to the MATE family of transporters. Antimicrob. Agents Chemother. 2005, 49, 4362-4364. [CrossRef] [PubMed]

195. Cho, Y.J.; Moon, D.C.; Jin, J.S.; Choi, C.H.; Lee, Y.C.; Lee, J.C. Genetic basis of resistance to aminoglycosides in Acinetobacter spp. and spread of armA in Acinetobacter baumannii sequence group 1 in Korean hospitals. Diagn. Microbiol. Infect. Dis. 2009, 64, 185-190. [CrossRef] [PubMed]

196. Yamane, K.; Wachino, J.; Doi, Y.; Kurokawa, H.; Arakawa, Y. Global spread of multiple aminoglycoside resistance genes. Emerg. Infect. Dis. 2005, 11, 951-953. [CrossRef]

197. Jana, S.; Deb, J.K. Molecular understanding of aminoglycoside action and resistance. Appl. Microbiol. Biotechnol. 2006, 70, 140-150. [CrossRef]

198. Zhu, J.; Wang, C.; Wu, J.; Jiang, R.; Mi, Z.; Huang, Z. A novel aminoglycoside-modifying enzyme gene aac $\left(6^{\prime}\right)-\mathrm{Ib}$ in a pandrug-resistant Acinetobacter baumannii strain. J. Hosp. Infect. 2009, 73, 184-185. [CrossRef]

199. Salimizand, H.; Zomorodi, A.R.; Mansury, D.; Khakshoor, M.; Azizi, O.; Khodaparast, S.; Baseri, Z.; Karami, P.; Zamanlou, S.; Farsiani, H.; et al. Diversity of aminoglycoside modifying enzymes and 16S rRNA methylases in Acinetobacter baumannii and Acinetobacter nosocomialis species in Iran; wide distribution of aadA1 and armA. Infect. Genet. Evol. 2018, 66, 195-199. [CrossRef]

200. Hasani, A.; Sheikhalizadeh, V.; Ahangarzadeh Rezaee, M.; Rahmati-Yamchi, M.; Hasani, A.; Ghotaslou, R.; Goli, H.R. Frequency of Aminoglycoside-Modifying Enzymes and ArmA Among Different Sequence Groups of Acinetobacter baumannii in Iran. Microb. Drug Resist. 2016, 22, 347-353. [CrossRef]

201. Doi, Y.; Adams, J.M.; Yamane, K.; Paterson, D.L. Identification of 16 S rRNA methylase-producing Acinetobacter baumannii clinical strains in North America. Antimicrob. Agents Chemother. 2007, 51, 4209-4210. [CrossRef]

202. Sheikhalizadeh, V.; Hasani, A.; Ahangarzadeh Rezaee, M.; Rahmati-Yamchi, M.; Hasani, A.; Ghotaslou, R.; Goli, H.R. Comprehensive study to investigate the role of various aminoglycoside resistance mechanisms in clinical isolates of Acinetobacter baumannii. J. Infect. Chemother. 2017, 23, 74-79. [CrossRef] [PubMed]

203. Appleman, M.D.; Belzberg, H.; Citron, D.M.; Heseltine, P.N.; Yellin, A.E.; Murray, J.; Berne, T.V. In vitro activities of nontraditional antimicrobials against multiresistant Acinetobacter baumannii strains isolated in an intensive care unit outbreak. Antimicrob. Agents Chemother. 2000, 44, 1035-1040. [CrossRef] [PubMed] 
204. Srinivasan, V.B.; Rajamohan, G.; Gebreyes, W.A. Role of AbeS, a novel efflux pump of the SMR family of transporters, in resistance to antimicrobial agents in Acinetobacter baumannii. Antimicrob. Agents Chemother. 2009, 53, 5312-5316. [CrossRef] [PubMed]

205. Okada, U.; Yamashita, E.; Neuberger, A.; Morimoto, M.; van Veen, H.W.; Murakami, S. Crystal structure of tripartite-type ABC transporter MacB from Acinetobacter baumannii. Nat. Commun. 2017, 8, 1336. [CrossRef] [PubMed]

206. Hameed, F.; Khan, M.A.; Muhammad, H.; Sarwar, T.; Bilal, H.; Rehman, T.U. Plasmid-mediated mcr-1 gene in Acinetobacter baumannii and Pseudomonas aeruginosa: First report from Pakistan. Rev. Soc. Bras. Med. Trop. 2019, 52, e20190237. [CrossRef] [PubMed]

207. Lima, W.G.; Alves, M.C.; Cruz, W.S.; Paiva, M.C. Chromosomally encoded and plasmid-mediated polymyxins resistance in Acinetobacter baumannii: A huge public health threat. Eur. J. Clin. Microbiol. Infect. Dis. 2018, 37, 1009-1019. [CrossRef]

208. Moffatt, J.H.; Harper, M.; Boyce, J.D. Mechanisms of Polymyxin Resistance. Adv. Exp. Med. Biol. 2019, 1145, 55-71.

209. Cheah, S.-E.; Johnson, M.D.; Zhu, Y.; Tsuji, B.T.; Forrest, A.; Bulitta, J.B.; Boyce, J.D.; Nation, R.L.; Li, J. Polymyxin Resistance in Acinetobacter baumannii: Genetic Mutations and Transcriptomic Changes in Response to Clinically Relevant Dosage Regimens. Sci. Rep. 2016, 6, 26233. [CrossRef]

210. Trebosc, V.; Gartenmann, S.; Tötzl, M.; Lucchini, V.; Schellhorn, B.; Pieren, M.; Lociuro, S.; Gitzinger, M.; Tigges, M.; Bumann, D.; et al. Dissecting Colistin Resistance Mechanisms in Extensively Drug-Resistant Acinetobacter baumannii Clinical Isolates. mBio 2019, 10, e01083-19. [CrossRef]

211. Moffatt, J.H.; Harper, M.; Harrison, P.; Hale, J.D.F.; Vinogradov, E.; Seemann, T.; Henry, R.; Crane, B.; St Michael, F.; Cox, A.D.; et al. Colistin resistance in Acinetobacter baumannii is mediated by complete loss of lipopolysaccharide production. Antimicrob. Agents Chemother. 2010, 54, 4971-4977. [CrossRef]

212. Whitfield, C.; Trent, M.S. Biosynthesis and export of bacterial lipopolysaccharides. Annu. Rev. Biochem. 2014, 83, 99-128. [CrossRef] [PubMed]

213. Hood, M.I.; Becker, K.W.; Roux, C.M.; Dunman, P.M.; Skaar, E.P. genetic determinants of intrinsic colistin tolerance in Acinetobacter baumannii. Infect. Immun. 2013, 81, 542-551. [CrossRef] [PubMed]

214. Liu, Y.-Y.; Wang, Y.; Walsh, T.R.; Yi, L.-X.; Zhang, R.; Spencer, J.; Doi, Y.; Tian, G.; Dong, B.; Huang, X.; et al. Emergence of plasmid-mediated colistin resistance mechanism MCR-1 in animals and human beings in China: A microbiological and molecular biological study. Lancet Infect. Dis. 2016, 16, 161-168. [CrossRef]

215. Meletis, G.; Skoura, L. Polymyxin Resistance Mechanisms: From Intrinsic Resistance to Mcr Genes. Recent Pat. Anti-Infect. Drug Discov. 2018, 13, 198-206. [CrossRef]

216. Martins-Sorenson, N.; Snesrud, E.; Xavier, D.E.; Cacci, L.C.; Iavarone, A.T.; McGann, P.; Riley, L.W.; Moreira, B.M. A novel plasmid-encoded mcr-4.3 gene in a colistin-resistant Acinetobacter baumannii clinical strain. J. Antimicrob. Chemother. 2020, 75, 60-64. [CrossRef]

(C) 2020 by the authors. Licensee MDPI, Basel, Switzerland. This article is an open access article distributed under the terms and conditions of the Creative Commons Attribution (CC BY) license (http://creativecommons.org/licenses/by/4.0/). 